\title{
Comparative performance of complex- valued $B$-spline and polynomial models applied to iterative frequency-domain decision feedback equalization of Hammerstein channels
}

Article

Accepted Version

Chen, S., Hong, X., Khalaf, E. F., Alsaadi, F. E. and Harris, C. J. (2017) Comparative performance of complex-valued Bspline and polynomial models applied to iterative frequencydomain decision feedback equalization of Hammerstein channels. IEEE Transactions on Neural Networks and Learning Systems, 28 (12). pp. 2872-2884. ISSN 2162-237X doi: https://doi.org/10.1109/TNNLS.2016.2609001 Available at https://centaur.reading.ac.uk/68339/

It is advisable to refer to the publisher's version if you intend to cite from the work. See Guidance on citing.

Published version at: http://dx.doi.org/10.1109/TNNLS.2016.2609001

To link to this article DOI: http://dx.doi.org/10.1109/TNNLS.2016.2609001

Publisher: IEEE Computational Intelligence Society

All outputs in CentAUR are protected by Intellectual Property Rights law, including copyright law. Copyright and IPR is retained by the creators or other copyright holders. Terms and conditions for use of this material are defined in 
the End User Agreement.

www.reading.ac.uk/centaur

\section{CentAUR}

Central Archive at the University of Reading

Reading's research outputs online 


\title{
Comparative Performance of Complex-Valued B-Spline and Polynomial Models Applied to Iterative Frequency-Domain Decision Feedback Equalization of Hammerstein Channels
}

\author{
Sheng Chen, Xia Hong, Emad Khalaf, Fuad E. Alsaadi and Chris J. Harris
}

\begin{abstract}
Complex-valued (CV) B-spline neural network approach offers a highly effective means for identifying and inverting practical Hammerstein systems. Compared to its conventional CV polynomial based counterpart, CV B-spline neural network has superior performance in identifying and inverting CV Hammerstein systems, while imposing a similar complexity. This paper reviews the optimality of $\mathrm{CV} B$-spline neural network approach. Advantages of B-spline neural network approach as compared to polynomial based modeling approach are extensively discussed, and the effectiveness of $\mathrm{CV}$ neural network based approach is demonstrated in a real-world application. More specifically, we evaluate the comparative performance of the $\mathrm{CV} B$-spline and polynomial based approaches for the nonlinear iterative frequency-domain decision feedback equalization (NIFDDFE) of single-carrier Hammerstein channels. Our results confirm the superior performance of the CV B-spline based NIFDDFE over its $\mathrm{CV}$ polynomial based counterpart.
\end{abstract}

Index Terms-Complex-valued (CV) B-spline neural network, $\mathrm{CV}$ polynomial model, identification and inversion of Hammerstein channels, nonlinear iterative frequency-domain decision feedback equalization

\section{INTRODUCTION}

In many real-world applications, the underlying system that generates complex-valued $(\mathrm{CV})$ signals can be modeled by the CV Hammerstein model. The system is grey-box, as its structure is known to be consisting of an unknown static nonlinearity followed by an unknown linear dynamic model. A well-known example of $\mathrm{CV}$ Hammerstein systems is the single-carrier (SC) block transmission communication channel with nonlinear high power amplifier (HPA) at transmitter, whereby the CV static nonlinearity of the Hammerstein system is constituted by the nonlinear transmit HPA, and its linear dynamic subsystem is the dispersive channel which can usually be modeled as a finite-duration impulse response (FIR) filter. Effective identification and inversion of CV Hammerstein systems is therefore crucial in these practical applications.

CV B-spline neural network has widely been used as an effective means for identification and inversion of $\mathrm{CV}$

S. Chen and C.J. Harris (sqc@ecs.soton.ac.uk, cjh@ecs.soton.ac.uk) are with Electronics and Computer Science, University of Southampton, Southampton SO17 1BJ, UK. S. Chen is also with King Abdulaziz University, Jeddah 21589, Saudi Arabia.

X. Hong (x.hong@ reading.ac.uk) is with Department of Computer Science, School of Mathematical, Physical and Computational Sciences, University of Reading, Reading, RG6 6AY, UK.

E. Khalaf and F.E. Alsaadi (ekhalaf@kau.edu.sa, fuad_alsaadi@yahoo.com) are with Electrical and Computer Engineering Department, Faculty of Engineering, King Abdulaziz University, Jeddah 21589, Saudi Arabia.

This project was funded by the Deanship of Scientific Research (DSR), King Abdulaziz University.
Hammerstein systems [1]-[3]. Compared to its conventional polynomial based counterpart, B-spline models are proven to have the optimal stability or numerical robustness [4][6], and achieve superior performance in challenging practical applications [1]-[3], while maintaining a similar computational complexity. In this paper, we review the $\mathrm{CV}$ B-spline neural network model as an effective means for identifying and inverting practical Hammerstein systems. In particular, we analyze its optimal robustness property, and provide the computational complexity required to calculate the output of a B-spline model, which turns out to be slightly higher than that of the conventional polynomial model, and the both models have the same order of complexity.

Our main contribution is however the derivation of a new CV B-spline neural network based design for the nonlinear iterative frequency-domain decision feedback equalization (NIFDDFE) of SC Hammerstein communication systems. Effective identification and inverting algorithms are provided for the SC Hammerstein channel based on the CV B-spline neural network approach. We use this challenging real-world application to evaluate the comparative performance of the CV B-spline neural network based NIFDDFE and its CV polynomial based NIFDDFE counterpart. The results obtained clearly demonstrate that our B-spline based NIFDDFE has a superior performance over the polynomial based NIFDDFE. Our novel application therefore reinforces the CV B-spline neural network as a versatile and effective means for solving real-world applications where the underlying systems can be represented by $\mathrm{CV}$ Hammerstein models.

Throughout this contribution, a $\mathrm{CV}$ number $x \in \mathbb{C}$ is represented either by $x=x_{R}+\mathrm{j} x_{I}$ or by $x=|x| \exp \left(\mathrm{j} \angle^{x}\right)$. The transpose and conjugate transpose operators are denoted by ()$^{\mathrm{T}}$ and ()$^{\mathrm{H}}$, respectively, while ()$^{-1}$ stands for the inverse operation and ()$^{*}$ denotes the conjugate operation. Furthermore, the expectation operator is denoted by $\mathrm{E}\{\}$.

\section{NIFDDFE FOR HAMMERSTEIN CHANNELS}

To illustrate the necessity for identifying and inverting CV Hammerstein systems, we begin by introducing our challenging application senario, the SC block transmission communication system [7]-[10], where each transmit block consists of $N$ data symbols with $M$-quadrature amplitude modulation (QAM) expressed as

$$
\boldsymbol{x}=\left[\begin{array}{llll}
x_{0} & x_{1} \cdots x_{N-1}
\end{array}\right]^{\mathrm{T}},
$$




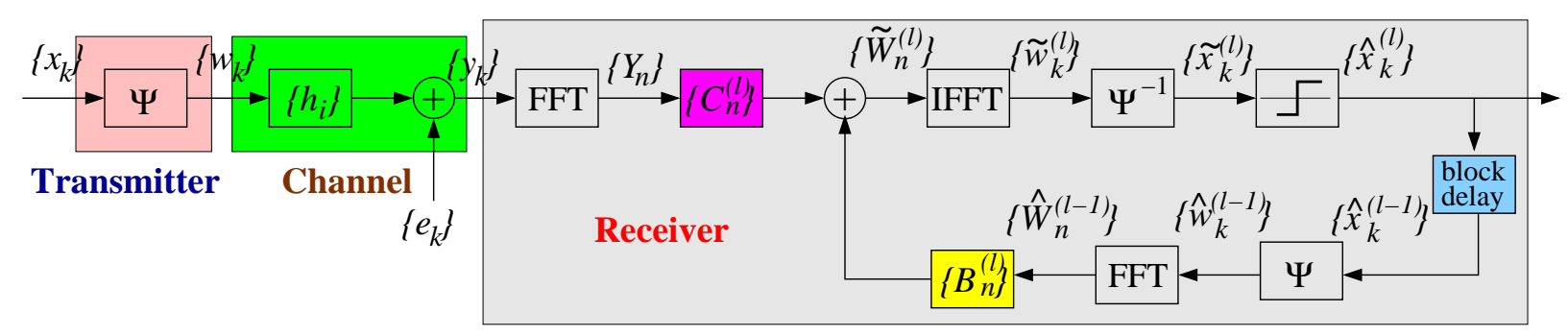

Fig. 1. System schematic of the NIFDDFE for SC Hammerstein communication systems with the nonlinear HPA $\Psi$ at transmitter.

where $x_{k}, 0 \leq k \leq N-1$, take the values from the $M$-QAM symbol set

$\mathbb{X}=\{d(2 l-\sqrt{M}-1)+\mathbf{j} \cdot d(2 q-\sqrt{M}-1), 1 \leq l, q \leq \sqrt{M}\}$,

with $2 d$ denoting the minimum distance between symbol points. Adding the cyclic prefix (CP) of length $N_{\text {cp }}$ to $\boldsymbol{x}$ yields

$$
\overline{\boldsymbol{x}}=\left[x_{-N_{\mathrm{cp}}} x_{-N_{\mathrm{cp}}+1} \cdots x_{-1} \mid \boldsymbol{x}^{\mathrm{T}}\right]^{\mathrm{T}},
$$

with $x_{-k}=x_{N-k}$ for $1 \leq k \leq N_{\mathrm{cp}}$. The signal block $\overline{\boldsymbol{x}}$ is amplified by the HPA to yield the transmitted signal block

$$
\overline{\boldsymbol{w}}=\left[w_{-N_{\mathrm{cp}}} w_{-N_{\mathrm{cp}}+1} \cdots w_{-1} \mid \boldsymbol{w}^{\mathrm{T}}\right]^{\mathrm{T}}
$$

where $\boldsymbol{w}=\left[\begin{array}{lll}w_{0} & w_{1} \cdots w_{N-1}\end{array}\right]^{\mathrm{T}}$ and

$$
w_{k}=\Psi\left(x_{k}\right),-N_{\mathrm{cp}} \leq k \leq N-1,
$$

in which $\Psi()$ represents the CV static nonlinearity of HPA, and $w_{-k}=w_{N-k}$ for $1 \leq k \leq N_{\mathrm{cp}}$. Typical HPA in transmitter is the solid state power amplifier [11]-[13], whose nonlinearity $\Psi($ ) is constituted by the HPA's amplitude response $A(r)$ and phase response $\Upsilon(r)$ given by

$$
\begin{aligned}
& A(r)=\frac{g_{a} r}{\left(1+\left(\frac{g_{a} r}{A_{\text {sat }}}\right)^{2 \beta_{a}}\right)^{\frac{1}{2 \beta_{a}}}}, \\
& \Upsilon(r)=\frac{\alpha_{\phi} r^{q_{1}}}{1+\left(\frac{r}{\beta_{\phi}}\right)^{q_{2}}},
\end{aligned}
$$

where $r$ denotes the amplitude of the input to HPA, $g_{a}$ is the small gain signal, $\beta_{a}$ is the smoothness factor and $A_{\text {sat }}$ is the saturation level, while the phase response parameters, $\alpha_{\phi}$. $\beta_{\phi}, q_{1}$ and $q_{2}$, are adjusted to match the specific amplifier's characteristics. We adopt the following parameter set defined in the standardization [12], [13]

$$
\begin{aligned}
& g_{a}=19, \beta_{a}=0.81, A_{\text {sat }}=1.4 ; \\
& \alpha_{\phi}=-48000, \beta_{\phi}=0.123, q_{1}=3.8, q_{2}=3.7 .
\end{aligned}
$$

Given the input $x_{k}=\left|x_{k}\right| e^{\mathrm{j} \angle^{x_{k}}}$, the output of the HPA is

$$
w_{k}=A\left(\left|x_{k}\right|\right) e^{\mathrm{j}\left(\angle^{x_{k}}+\Upsilon\left(\left|x_{k}\right|\right)\right)} .
$$

The operating status of the HPA is specified by the output back-off $(\mathrm{OBO})$, which is defined as the ratio of the maximum output power $P_{\max }$ of the HPA to the average output power $P_{\text {aop }}$ of the HPA output signal, given by

$$
\text { OBO }=10 \cdot \log _{10} \frac{P_{\max }}{P_{\text {aop }}} .
$$

The smaller OBO is, the more the HPA is operating into the nonlinear saturation region.
The amplified signal block $\overline{\boldsymbol{w}}$ is transmitted through the channel whose channel impulse response (CIR) coefficient vector is

$$
\boldsymbol{h}=\left[h_{0} h_{1} \cdots h_{L_{\mathrm{cir}}}\right]^{\mathrm{T}},
$$

where $L_{\text {cir }}$ denotes the CIR length. Note that the CP must be chosen to be $N_{\mathrm{cp}} \geq L_{\mathrm{cir}}$. We can always assume that $h_{0}=1$ because if this is not the case, $h_{0}$ can be absorbed into the CV nonlinearity $\Psi($ ), and the CIR coefficients are rescaled as $h_{i} / h_{0}$ for $0 \leq i \leq L_{\text {cir. }}$. The combined transmission channel and transmitter, as seen in Fig. 1, is a Hammerstein system containing the nonlinearity $\Psi($ ) defined by (6) and (7) followed by the FIR filter with the CIR (11).

At receiver, after $\mathrm{CP}$ removal, the channel-impaired received signals $y_{k}$ are given by

$$
y_{k}=\sum_{i=0}^{L_{\mathrm{cir}}} h_{i} w_{k-i}+e_{k}, 0 \leq k \leq N-1,
$$

in which $w_{k-i}=w_{N+k-i}$ for $k<i$, where $e_{k}$ is the additive white Gaussian noise (AWGN) with $E\left\{\left|e_{k}\right|^{2}\right\}=2 \sigma_{e}^{2}$. Our NIFDDFE receiver is depicted in Fig. 1. First, passing $\boldsymbol{y}=$ $\left[\begin{array}{llll}y_{0} & y_{1} & \cdots & y_{N-1}\end{array}\right]^{\mathrm{T}}$ through the $N$-point fast Fourier transform (FFT) processor yields the frequency-domain (FD) received signal block $\boldsymbol{Y}=\left[\begin{array}{llll}Y_{0} & Y_{1} & \cdots & Y_{N-1}\end{array}\right]^{\mathrm{T}}$ with elements

$$
Y_{n}=\sum_{k=0}^{N-1} y_{k} e^{-\mathrm{j} \frac{2 \pi k n}{N}}, 0 \leq n \leq N-1
$$

Due to the well-known circular property of CP [7]-[10],

$$
Y_{n}=H_{n} W_{n}+\Xi_{n}, 0 \leq n \leq N-1,
$$

in which $\Xi_{n}$ is the FD representation of the AWGN with $\mathrm{E}\left\{\left|\Xi_{n}\right|^{2}\right\}=2 \sigma_{e}^{2}$, and $\boldsymbol{W}=\left[\begin{array}{ll}W_{0} & W_{1} \cdots W_{N-1}\end{array}\right]^{\mathrm{T}}$ is the $N$-point FFT of $\boldsymbol{w}$, i.e.,

$$
W_{n}=\sum_{k=0}^{N-1} w_{k} e^{-\mathrm{j} \frac{2 \pi k n}{N}}, 0 \leq n \leq N-1,
$$

with $\mathrm{E}\left\{\left|W_{n}\right|^{2}\right\}=N \mathrm{E}\left\{\left|w_{k}\right|^{2}\right\}=N \sigma_{w}^{2}$, while the FD channel transfer function coefficients (FDCTFCs) $H_{n}, 0 \leq$ $n \leq N-1$, are the $N$-point FFT of $\boldsymbol{h}$ given by

$$
H_{n}=\sum_{i=0}^{L_{\mathrm{cir}}} h_{i} e^{-\mathrm{j} \frac{2 \pi i n}{N}}, 0 \leq n \leq N-1 .
$$

Our new NIFDDFE involves an iterative detection procedure with the iteration index $l \geq 1$. Typically 3 to 4 iterations are sufficient. Specifically, let the FD feedforward and feedback equalizers coefficients at the $l$ th iteration by $\left\{C_{n}^{(l)}\right\}_{n=0}^{N-1}$ 
and $\left\{B_{n}^{(l)}\right\}_{n=0}^{N-1}$, respectively. Further denote the estimate of $\left\{W_{n}\right\}_{n=0}^{N-1}$ at the previous iteration be $\left\{\widehat{W}_{n}^{(l-1)}\right\}_{n=0}^{N-1}$. Then the 'soft' estimate of $W_{n}$ is given by

$$
\widetilde{W}_{n}^{(l)}=C_{n}^{(l)} Y_{n}+B_{n}^{(l)} \widehat{W}_{n}^{(l-1)}, 0 \leq n \leq N-1 .
$$

Passing $\widetilde{W}_{n}^{(l)}$ for $0 \leq n \leq N-1$ through the $N$-point inverse FFT (IFFT) processor yields the soft estimate of the timedomain (TD) transmitted signals $\left\{w_{k}\right\}_{k=0}^{N-1}$ as

$$
\widetilde{w}_{k}^{(l)}=\frac{1}{N} \sum_{n=0}^{N-1} \widetilde{W}_{n}^{(l)} e^{\mathrm{j} \frac{2 \pi n k}{N}}, 0 \leq k \leq N-1 .
$$

For the convenience of discussion, assume that the nonlinearity $\Psi\left(\right.$ ) of the transmitter HPA and its inversion $\Psi^{-1}()$ are both known at the receiver. The soft estimate $\left\{\widetilde{x}_{k}^{(l)}\right\}_{k=0}^{N-1}$ of the transmitted data symbols can be calculated according to

$$
\widetilde{x}_{k}^{(l)}=\Psi^{-1}\left(\widetilde{w}_{k}^{(l)}\right), 0 \leq k \leq N-1 .
$$

By quantizing $\widetilde{x}_{k}^{(l)}$, we obtain the hard-decision estimate $\left\{\widehat{x}_{k}^{(l)}\right\}_{k=0}^{N-1}$ of the transmitted data block. Further distorting $\left\{\widehat{x}_{k}^{(l)}\right\}_{k=0}^{N=0}$ by $\Psi()$ yields the TD estimate $\left\{\widehat{w}_{k}^{(l)}\right\}_{k=0}^{N-1}$ which is transformed by the $N$-point FFT to produce the FD estimate $\left\{\widehat{W}_{n}^{(l)}\right\}_{k=0}^{N-1}$ to be used in the next iteration.

If the HPA is linear and hence $w_{k}=x_{k}$, we have the existing linear iterative FD decision feedback equalisation (LIFDDFE), for which $\left\{C_{n}^{(l)}\right\}_{n=0}^{N-1}$ and $\left\{B_{n}^{(l)}\right\}_{n=0}^{N-1}$ can be obtained by minimizing the mean square error but the computation is quite involved [8]. Extending this LIFDDFE design to our new NIFDDFE also yields poor performance. However, we find that the extension of the low-complexity simplified LIFDDFE design of [10] to our NIFDDFE works well with some modifications. We now present how to calculate $\left\{C_{n}^{(l)}\right\}_{n=0}^{N-1}$ and $\left\{B_{n}^{(l)}\right\}_{n=0}^{N-1}$ for our new NIFDDFE.

At the first iteration $l=1, \widehat{W}_{n}^{(0)}=0$ and $B_{n}^{(1)}=0$ for $0 \leq n \leq N-1$, and we have

$$
C_{n}^{(1)}=\frac{H_{n}^{*}}{\left|H_{n}\right|^{2}+\frac{2 \sigma_{e}^{2}}{\sigma_{w}^{2}}}, 0 \leq n \leq N-1,
$$

which is identical to the nonlinear FD equalization (NFDE) solution of [3]. For the iterations $l \geq 2$, we have

$$
\begin{aligned}
& C_{n}^{(l)}=C_{n}=\frac{(1-\gamma) H_{n}^{*}}{\operatorname{SNR}_{\mathrm{pre}}^{-1}+\beta P_{\mathrm{e}, \mathrm{pre}}\left|H_{n}\right|^{2}}, 0 \leq n \leq N-1, \\
& B_{n}^{(l)}=B_{n}=-\left(C_{n} H_{n}-1\right), 0 \leq n \leq N-1,
\end{aligned}
$$

with

$$
\begin{aligned}
& \varpi=\frac{1}{N} \sum_{n=0}^{N-1} \frac{\left|H_{n}\right|^{2}}{\mathrm{SNR}_{\mathrm{pre}}^{-1}+\beta P_{\mathrm{e}, \mathrm{pre}}\left|H_{n}\right|^{2}}, \\
& \gamma=\frac{\varpi}{1+\varpi} .
\end{aligned}
$$

For the LIFDDFE, the work [10] finds that the performance is insensitive to the predefined SNR value $\mathrm{SNR}_{\text {pre }}$ and the predefined symbol error probability $P_{\mathrm{e}, \mathrm{pre}}$. In particular, $\mathrm{SNR}_{\mathrm{pre}}^{-1}=0.1$ and $P_{\mathrm{e}, \mathrm{pre}}=0.1$ yields excellent results. In our NIFDDFE, we also find that $\mathrm{SNR}_{\mathrm{pre}}^{-1}=0.1$ and $P_{\mathrm{e}, \mathrm{pre}}=0.1$ are appropriate. In the LIFDDFE case, i.e., $w_{k}=x_{k}, \beta$ is a parameter depending on the modulation scheme for $x_{k}$. Specifically, $\beta=2,2 / 5$ and $2 / 21$ for $4-$ QAM, 16-QAM and 64-QAM, respectively. In our NIFDDFE, $w_{k}$ is a nonlinearly distorted $x_{k}$ and the severity of this nonlinear distortion depends on the $\mathrm{OBO}$ of the transmitter HPA. Intuitively, $\beta$ should be smaller than the linear case and how small $\beta$ is also depends on the value of OBO. For 64QAM with $\mathrm{OBO}=3 \mathrm{~dB}$, we find $\beta=0.01$ is appropriate, i.e., ten times smaller than the linear case. With $\mathrm{OBO}=5 \mathrm{~dB}$, an appropriate value is $\beta=0.05$, i.e., only two times smaller than the linear case. This makes sense, as with $\mathrm{OBO}=5 \mathrm{~dB}$, the HPA is operating closer to the linear region than the case of $\mathrm{OBO}=3 \mathrm{~dB}$. Another modification made is in the feedback coefficients $B_{n}$ of (22). In the LIFDDFE design [10], $B_{n}=-\left(C_{n} H_{n}-\gamma\right)$. But we find that with $B_{n}$ of (22), the performance is better for the NIFDDFE.

\section{CV B-Spline AND POLYNOMIAL IMPLEMENTATIONS OF NIFDDFE}

It can be seen that implementing the NIFDDFE requires to identifying and inverting the Hammerstein channel that consists of the unknown static nonlinearity $\Psi($ ) followed by the FIR filter with the unknown CIR vector $\boldsymbol{h}$.

\section{A. CV B-spline and polynomial models for $\Psi($ )}

1) CV B-spline neural network: The CV B-spline neural network approach [1]-[3] offers an effective means for identifying and inverting this Hammerstein channel. We first point out that $\Psi($ ) meets the following conditions.

i) $\Psi($ ) is a one-to-one mapping, i.e., a continuous and invertible function.

ii) $x_{R}$ and $x_{I}$ are upper and lower bounded by some known finite real values, where $x=x_{R}+\mathrm{j} x_{I}$ denotes the $\mathrm{CV}$ input to $\Psi\left(\right.$ ), and the distributions of $x_{R}$ and $x_{I}$ are identical.

According to the property ii), we have $U_{\min }<x_{\mathrm{s}}<U_{\max }$, where $U_{\min }$ and $U_{\max }$ are known finite real values, while $x_{\mathrm{s}}$ denotes either $x_{R}$ or $x_{I}$, i.e., the subscript $\mathrm{s}$ is either $R$ or $I$. To use a B-spline neural network for modeling $\Psi($ ), a set of $N_{\mathrm{s}}$ univariate B-spline basis functions on $x_{\mathrm{s}}$ is parametrized by the piecewise polynomial degree $P_{o}$ and a knot sequence of $\left(N_{\mathrm{s}}+P_{o}+1\right)$ knot values, $\left\{U_{0}, U_{1}, \cdots, U_{N_{\mathrm{s}}+P_{o}}\right\}$, with

$$
\begin{gathered}
U_{0}<U_{1}<\cdots<U_{P_{o}-2}<U_{P_{o}-1}=U_{\min }<U_{P_{o}}<\cdots< \\
U_{N_{\mathrm{s}}}<U_{N_{\mathrm{s}}+1}=U_{\max }<U_{N_{\mathrm{s}}+2}<\cdots<U_{N_{\mathrm{s}}+P_{o}} .
\end{gathered}
$$

At each end, there are $P_{o}-1$ 'external' knots that are outside the input region and one boundary knot. As a result, the number of 'internal' knots is $N_{\mathrm{s}}+1-P_{o}$. Given the set of predetermined knots (25), the set of $N_{\mathrm{s}}$ B-spline basis functions can be formed by using the De Boor recursion [14], yielding for $1 \leq l \leq N_{\mathrm{s}}+P_{o}$,

$$
B_{l}^{(\mathrm{s}, 0)}\left(x_{\mathrm{s}}\right)= \begin{cases}1, & \text { if } U_{l-1} \leq x_{\mathrm{s}}<U_{l} \\ 0, & \text { otherwise }\end{cases}
$$

as well as for $l=1, \cdots, N_{\mathrm{s}}+P_{o}-p$ and $p=1, \cdots, P_{o}$,

$$
\begin{aligned}
B_{l}^{(\mathrm{s}, p)}\left(x_{\mathrm{s}}\right)= & \frac{x_{\mathrm{s}}-U_{l-1}}{U_{p+l-1}-U_{l-1}} B_{l}^{(\mathrm{s}, p-1)}\left(x_{\mathrm{s}}\right) \\
& +\frac{U_{p+l}-x_{\mathrm{s}}}{U_{p+l}-U_{l}} B_{l+1}^{(\mathrm{s}, p-1)}\left(x_{\mathrm{s}}\right) .
\end{aligned}
$$


De Boor recursion is illustrated in Fig. 2.

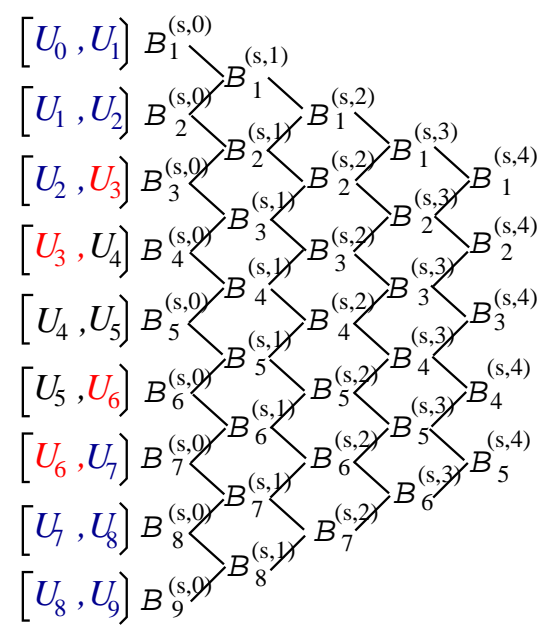

Fig. 2. De Boor recursion: $P_{o}=4, N_{\mathrm{s}}=5, U_{\min }=U_{3}$ and $U_{\max }=U_{6}$.

Using the tensor product between the two sets of univariate B-spline basis functions [15], $B_{l}^{\left(R, P_{o}\right)}\left(x_{R}\right)$ for $1 \leq l \leq N_{R}$ and $B_{m}^{\left(I, P_{o}\right)}\left(x_{I}\right)$ for $1 \leq m \leq N_{I}$, a set of new B-spline basis functions $B_{l, m}^{\left(P_{o}\right)}(x)$ can be formed and used in the $\mathrm{CV}$ $\mathrm{B}$-spline neural network, giving rise to

$$
\begin{aligned}
\widehat{w} & =\widehat{\Psi}_{B}(x)=\sum_{l=1}^{N_{R}} \sum_{m=1}^{N_{I}} B_{l, m}^{\left(P_{o}\right)}(x) \theta_{l, m}^{B} \\
& =\sum_{l=1}^{N_{R}} \sum_{m=1}^{N_{I}} B_{l}^{\left(R, P_{o}\right)}\left(x_{R}\right) B_{m}^{\left(I, P_{o}\right)}\left(x_{I}\right) \theta_{l, m}^{B},
\end{aligned}
$$

where $\theta_{l, m}^{B}=\theta_{l, m_{R}}^{B}+\mathrm{j} \theta_{l, m_{I}}^{B} \in \mathbb{C}, 1 \leq l \leq N_{R}$ and $1 \leq m \leq$ $N_{I}$, are the $\mathrm{CV}$ weights. Denote

$$
\boldsymbol{\theta}_{B}=\left[\theta_{1,1}^{B} \theta_{1,2}^{B} \cdots \theta_{l, m}^{B} \cdots \theta_{N_{R}, N_{I}}^{B}\right]^{\mathrm{T}} \in \mathbb{C}^{N_{B}},
$$

where $N_{B}=N_{R} N_{I}$. The task of identifying the nonlinearity $\Psi\left(\right.$ ) is turned into one of estimating $\boldsymbol{\theta}_{B}$.

2) $C V$ polynomial model: Similarly for the conventional polynomial modeling with polynomial degree $P_{o}$, let us define the set of $P_{o}+1$ polynomial basis functions as

$$
P_{l}^{(\mathrm{s})}\left(x_{\mathrm{s}}\right)=x_{\mathrm{s}}^{l}, 0 \leq l \leq P_{o} .
$$

Then using the tensor product between the two sets of univariate polynomial basis functions, $P_{l}^{(R)}\left(x_{R}\right)$ for $0 \leq l \leq P_{o}$ and $P_{m}^{(I)}\left(x_{I}\right)$ for $0 \leq m \leq P_{o}$, a set of new polynomial basis functions $P_{l, m}(x)=P_{l}^{(\bar{R})}\left(x_{R}\right) P_{m}^{(I)}\left(x_{I}\right)$ for $0 \leq l, m \leq P_{o}$ can be formed, giving rise to the $\mathrm{CV}$ polynomial model

$$
\begin{aligned}
\widehat{w} & =\widehat{\Psi}_{P}(x)=\sum_{l=0}^{P_{o}} \sum_{m=0}^{P_{o}} P_{l, m}(x) \theta_{l, m}^{P} \\
& =\sum_{l=0}^{P_{o}} \sum_{m=0}^{P_{o}} P_{l}^{(R)}\left(x_{R}\right) P_{m}^{(I)}\left(x_{I}\right) \theta_{l, m}^{P},
\end{aligned}
$$

where $\theta_{l, m}^{P}=\theta_{l, m_{R}}^{P}+\mathrm{j} \theta_{l, m_{I}}^{P} \in \mathbb{C}, 0 \leq l, m \leq P_{o}$, are the $\mathrm{CV}$ weights. Define

$$
\boldsymbol{\theta}_{P}=\left[\theta_{0,0}^{P} \theta_{0,1}^{P} \cdots \theta_{l, m}^{P} \cdots \theta_{P_{o}, P_{o}}^{P}\right]^{\mathrm{T}} \in \mathbb{C}^{N_{P}},
$$

where $N_{P}=\left(1+P_{o}\right)^{2}$. The task of identifying the nonlinearity $\Psi\left(\right.$ ) becomes one of estimating $\boldsymbol{\theta}_{P}$.

\section{B. Model structure parameters}

1) Polynomial model: For the conventional polynomial model, there is only one model structure parameter, and choosing the polynomial degree $P_{o}=4$ is sufficient for most practical applications.

2) B-spline model: For the B-spline neural network, choosing $P_{o}=4$ is also sufficient for most applications. In our application, the knot sequence is symmetric and $U_{\min }=-U_{\max }$. Given the required average transmitted signal power, the peak amplitude in the symbol set (2) is known and hence $U_{\max }$ is known. $N_{R}=N_{I}=N_{\mathrm{s}}=6$ to 10 is sufficient for accurately modeling on the finite interval $\left[U_{\min }, U_{\max }\right]$. The $N_{\mathrm{s}}+1-P_{o}$ internal knots may be uniformly spaced in the interval $\left[U_{\min }, U_{\max }\right]$. Note that there exist no data for $x_{\mathrm{s}}<U_{\min }$ and $x_{\mathrm{s}}>U_{\max }$ in identification but it is desired that the B-spline model has certain extrapolating capability outside the interval $\left[U_{\min }, U_{\max }\right]$. The external knots can be set empirically to meet the required extrapolation capability. However, the precise choice of these external knots does not really matter, in terms of modeling accuracy.

\section{Complexity analysis}

1) Complexity of polynomial model (31): Complexity analysis of the $\mathrm{CV}$ polynomial model is straightforward, and the computational complexity of computing the polynomial model (31) is obviously on the order of $\left(1+P_{o}\right)^{2}$, denoted as $\mathrm{O}\left(\left(P_{o}+1\right)^{2}\right)$. As an example, the computational requirements for $P_{o}=4$ are listed in Table I.

TABLE I

COMPLEXITY OF POLYNOMIAL MODEL (31) FOR $P_{o}=4$.

\begin{tabular}{c|cc}
\hline Computation & Multiplications & Additions \\
\hline Two sets of 1-D basis functions & $2 \times 4$ & 0 \\
Output of (31) & $3 \times 25$ & $2 \times 24$ \\
\hline Total & 83 & 48 \\
\hline
\end{tabular}

2) Complexity of B-spline model (28): Comparing the Bspline modeling of (26) to (28) with the polynomial modeling of (30) and (31) and noting that $N_{B}$ can be significantly larger than $N_{P}$, it would appear that the complexity of the CV Bspline model would be significantly higher than that of the $\mathrm{CV}$ polynomial model. This is in fact not the case, and the complexity of the $\mathrm{CV}$ B-spline modeling also depends only on $P_{o}$, not the number of basis functions $N_{\mathrm{s}}$.

Given $x_{\mathrm{s}} \in\left[U_{\min }, U_{\max }\right]$, there are only $P_{o}+1$ basis functions with nonzero values at most. Fig. 3 illustrates the complexity of generating the B-spline basis function set for $P_{o}=4$, which shows that the total requirements are 26

TABLE II

COMPLEXITY OF B-SPLINE MODEL (28) FOR $P_{o}=4$.

\begin{tabular}{l|cc}
\hline Computation & Multiplications & Additions \\
\hline Upper bound: & & \\
\hline Two sets of 1-D basis functions & $2 \times 38$ & $2 \times 26$ \\
Output of (28) & $3 \times 25$ & $2 \times 24$ \\
\hline Total & 151 & 100 \\
\hline Lower bound: & & \\
\hline Two sets of 1-D basis functions & $2 \times 36$ & $2 \times 25$ \\
Output of (28) & $3 \times 16$ & $2 \times 15$ \\
\hline Total & 120 & 80 \\
\hline
\end{tabular}




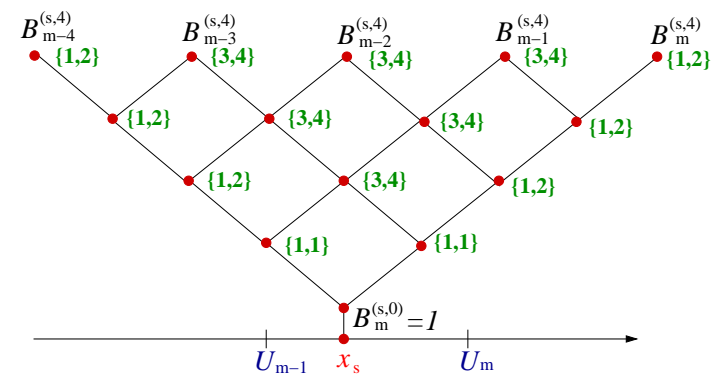

(a) $P_{o}+1 \leq m \leq N_{\mathrm{s}}$

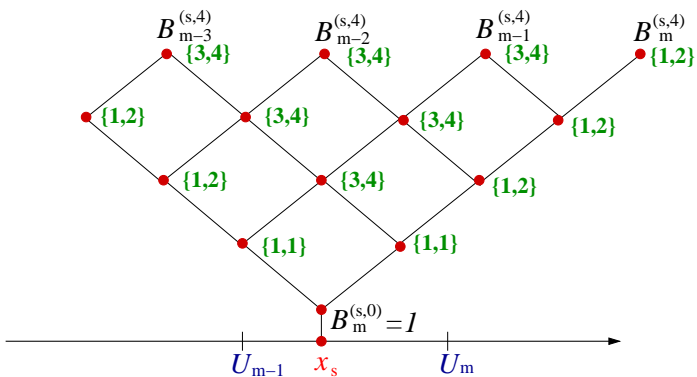

(b) $m=P_{o}$

Fig. 3. Complexity of B-spline model with $P_{o}=4$ using De Boor recursion, where $\{a, b\}$ beside a node indicates that it requires $a$ additions and $b$ multiplications to compute the basis function value at this node. The case of $m=N_{\mathrm{s}}+1$ is identical to (b).

additions and 38 multiplications at most. Thus, in the tensorproduct B-spline model (28), there are only $\left(P_{o}+1\right)^{2}$ nonzero basis functions at most for any given input, which is comparable to the tensor-product polynomial model (31) with $\left(P_{o}+1\right)^{2}$ non-zero basis functions. The upper-bound and lower-bound computational requirements for the CV B-spline modeling with $P_{o}=4$ are listed in Table II, where it can be seen that the complexity of the B-spline modeling is no more than twice of the polynomial modeling. Therefore, the computational complexity of computing the B-spline model (28) is still on the order of $\mathrm{O}\left(\left(P_{o}+1\right)^{2}\right)$.

\section{Optimal robustness property of B-spline model}

A critical aspect to consider in a model representation is its stability with respect to perturbation of the model parameters, because in identification, the data are inevitably noisy, which will perturb the model parameters away from their true values. A significant advantage of the B-spline model over the polynomial model is its superior numerical stability. B-spline functions are optimally stable bases [4]-[6], and this optimality is due to the convexity of its model bases, i.e., they are all positive and sum up to one. By contrast, the polynomial model is far inferior in terms of numerical stability.

Let us first analyze this aspect theoretically. Assume that the real-valued true system can be represented by the polynomial model of degree $P_{o}$ exactly as

$$
y_{\mathrm{s}}=\sum_{i=0}^{P_{o}} a_{i} x_{\mathrm{s}}^{i}
$$

as well as by the following B-spline model exactly

$$
y_{\mathrm{s}}=\sum_{i=1}^{N_{\mathrm{s}}} b_{i} B_{i}^{\left(\mathrm{s}, P_{o}\right)}\left(x_{\mathrm{s}}\right)
$$

where $y_{\mathrm{s}}, x_{\mathrm{s}} \in \mathbb{R}$. Because of the noisy identification data, the estimated model coefficients are perturbed from their true values to $\widehat{a}_{i}=a_{i}+\varepsilon_{i}$ for the polynomial model, and to $\widehat{b}_{i}=$ $b_{i}+\varepsilon_{i}$ for the B-spline model. Assume that all the estimation noises $\varepsilon_{i}$ are bounded by $\left|\varepsilon_{i}\right|<\varepsilon_{\max }$. The upper bound of $\left|y_{\mathrm{s}}-\widehat{y}_{\mathrm{s}}\right|$ for the B-spline model can be worked out to be

$$
\begin{aligned}
\left|y_{\mathrm{s}}-\widehat{y}_{\mathrm{s}}\right| & =\left|\sum_{i=1}^{N_{\mathrm{s}}} b_{i} B_{i}^{\left(\mathrm{s}, P_{o}\right)}\left(x_{\mathrm{s}}\right)-\sum_{i=1}^{N_{\mathrm{s}}} \widehat{b}_{i} B_{i}^{\left(\mathrm{s}, P_{o}\right)}\left(x_{\mathrm{s}}\right)\right| \\
& <\varepsilon_{\max }\left|\sum_{i=1}^{N_{\mathrm{s}}} B_{i}^{\left(\mathrm{s}, P_{o}\right)}\left(x_{\mathrm{s}}\right)\right|=\varepsilon_{\max }
\end{aligned}
$$

Observe that the upper bound of the B-spline model output perturbation only depends on the upper bound of the perturbation noise, and it does not depend on the input value $x_{\mathrm{S}}$, the number of basis functions $N_{\mathrm{s}}$ or the polynomial degree $P_{o}$. Hence, the B-spline model enjoys the maximum numerical robustness, and this optimal robustness property is well known. By contrast, the upper bound of $\left|y_{\mathrm{s}}-\widehat{y}_{\mathrm{s}}\right|$ for the polynomial model can be worked out to be

$$
\left|y_{\mathrm{s}}-\widehat{y}_{\mathrm{s}}\right|=\left|\sum_{i=0}^{P_{o}} a_{i} x_{\mathrm{s}}^{i}-\sum_{i=0}^{P_{o}} \widehat{a}_{i} x_{\mathrm{s}}^{i}\right|<\varepsilon_{\max }\left|\sum_{i=0}^{P_{o}} x_{\mathrm{s}}^{i}\right| .
$$

Observe that the upper bound of the polynomial model output perturbation depends not only on the upper bound of the perturbation noise but also on the input value $x_{\mathrm{s}}$ and the polynomial degree $P_{o}$. The higher the polynomial degree $P_{o}$, the more serious the polynomial model may be perturbed, a well-known drawback of using polynomial modeling.
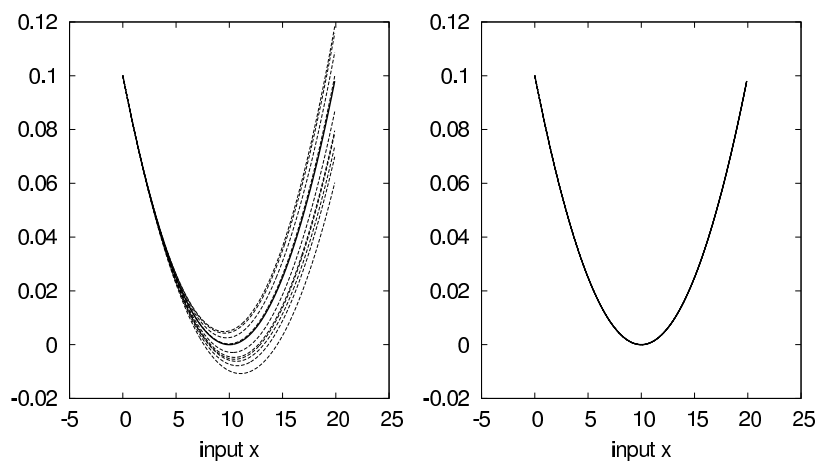

(a)

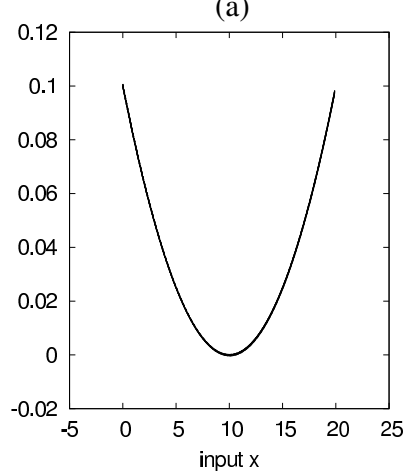

(c)

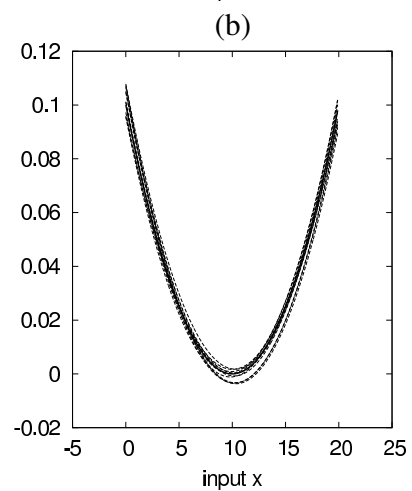

(d)
Fig. 4. (a) Polynomial model with UDRN perturbation noises drawn from $[-0.0001,0.0001]$, (b) B-spline model with UDRN perturbation noises drawn from $[-0.0001,0.0001]$, (c) B-spline model with UDRN perturbation noises drawn from $[-0.001,0.001]$, and (d) B-spline model with UDRN perturbation noises drawn from $[-0.01,0.01]$. Cited from [16]. 
We use the simple example of [16] to demonstrate the excellent numerical stability of the B-spline model over the polynomial model in Fig. 4. Fig. 4 (a) plots a quadratic polynomial function $y_{\mathrm{s}}=0.001 x_{\mathrm{s}}^{2}-0.02 x_{\mathrm{s}}+0.1$ defined over $x_{\mathrm{s}} \in[0,20]$ in solid curve. With the knot sequence $\{-5,-4,0,20,24,25\}$, this function is modeled as a quadratic $\mathrm{B}$-spline model of $y_{\mathrm{s}}=0.14 B_{1}^{(\mathrm{s}, 2)}\left(x_{\mathrm{s}}\right)-0.10 B_{2}^{(\mathrm{s}, 2)}\left(x_{\mathrm{s}}\right)+$ $0.14 B_{3}^{(\mathrm{s}, 2)}\left(x_{\mathrm{s}}\right)$, which is depicted in Fig. 4 (b) in solid curve. We draw three noises from a uniformly distributed random number (UDRN) in $[-0.0001,0.0001]$, and add them to the three parameters in the two models, respectively. Fig. 4 (a) and (b) depict the ten sets of the perturbed functions in dashed curve generated by perturbing the two models' parameters. It can be clearly seen from Fig. 4 (a) that the polynomial model is seriously perturbed, but there is no noticeable change at all in Fig. 4 (b) for the B-spline model. Next we draw three perturbation noises from a UDRN in [-0.001, 0.001], and add them to the three parameters of the B-spline model. Again, the B-spline model is hardly affected, as can be seen from Fig. 4 (c). We then draw three perturbation noises from a UDRN in $[-0.01,0.01]$ to add to the three B-spline parameters, and the results obtained are shown in Fig. 4 (d). Observe from Fig. 4 (a) and (d) that, despite of the fact that the strength of the perturbation noise added to the B-spline model coefficients is 100 times larger than that added to the polynomial model coefficients, the B-spline model is still much less seriously perturbed than the polynomial model.

\section{E. Identifying Hammerstein channel}

We will present the identification of the Hammerstein channel using the CV B-spline neural network approach, since the identification algorithm is identical using the $\mathrm{CV}$ polynomial modeling approach. Therefore, we drop the subscript $B$ and superscript ${ }^{B}$ from the B-spline model.

Given a block of $N$ training data, $\left\{x_{k}, y_{k}\right\}_{k=0}^{N-1}$, the identification task is to obtain the estimates of $\boldsymbol{h}$ and $\boldsymbol{\theta}$ by minimizing the cost function

$$
J(\boldsymbol{h}, \boldsymbol{\theta})=\frac{1}{N} \sum_{k=0}^{N-1}\left|\widehat{e}_{k}\right|^{2}=\frac{1}{N} \sum_{k=0}^{N-1}\left|y_{k}-\widehat{y}_{k}\right|^{2},
$$

subject to the constraint of $h_{0}=1$, in which the prediction of $y_{k}$ is given by

$$
\widehat{y}_{k}=\sum_{i=0}^{L_{\mathrm{cir}}} h_{i} \widehat{w}_{k-i}=\sum_{i=0}^{L_{\mathrm{cir}}} h_{i} \sum_{l=1}^{N_{R}} \sum_{m=1}^{N_{I}} B_{l, m}^{\left(P_{o}\right)}\left(x_{k-i}\right) \theta_{l, m},
$$

where $x_{k-i}=x_{N+k-i}$ if $k<i$. The cost function (33) is convex with respect to $\boldsymbol{h}$ when fixing $\boldsymbol{\theta}$, and convex with respect to $\boldsymbol{\theta}$ given $\boldsymbol{h}$. According to [17], [18], the estimates of $\boldsymbol{\theta}$ and $\boldsymbol{h}$ are unbiased, irrespective to the algorithm used to minimize the cost function (33). In [16], an alternating least squares (ALS) procedure was proposed which guarantees to find the unique optimal solution of $\boldsymbol{\theta}$ and $\boldsymbol{h}$ in only a few iterations. We adopt this ALS procedure in our current application. This ALS procedure is summarized below.

Initialisation. Define the amalgamated parameter vector

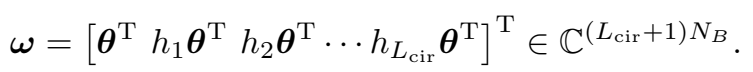

Further define the regression matrix $\boldsymbol{P} \in \mathbb{R}^{N \times\left(L_{\mathrm{cir}}+1\right) N_{B}}$

$$
\boldsymbol{P}=\left[\begin{array}{cccc}
\phi^{\mathrm{T}}(0) & \phi^{\mathrm{T}}(-1) & \cdots & \phi^{\mathrm{T}}\left(-L_{\text {cir }}\right) \\
\vdots & \vdots & \vdots & \vdots \\
\phi^{\mathrm{T}}(k) & \phi^{\mathrm{T}}(k-1) & \cdots & \phi^{\mathrm{T}}\left(k-L_{\text {cir }}\right) \\
\vdots & \vdots & \vdots & \vdots \\
\phi^{\mathrm{T}}(N-1) & \phi^{\mathrm{T}}(N-2) & \cdots & \phi^{\mathrm{T}}\left(N-1-L_{\text {cir }}\right)
\end{array}\right]
$$

with $\phi(k)=\left[\phi_{1,1}(k) \phi_{1,2}(k) \cdots \phi_{l, m}(k) \cdots \phi_{N_{R}, N_{I}}(k)\right]^{\mathrm{T}}$, in which $\phi_{l, m}(k)=B_{l, m}^{\left(P_{o}\right)}\left(x_{k}\right)$ for $1 \leq l \leq N_{R}$ and $1 \leq m \leq$ $N_{I}$. The regularized least squares (LS) estimate of $\boldsymbol{\omega}$ is $\widehat{\boldsymbol{\omega}}=$ $\left(\boldsymbol{P}^{\mathrm{T}} \boldsymbol{P}+\rho \boldsymbol{I}\right)^{-1} \boldsymbol{P}^{\mathrm{T}} \boldsymbol{y}$, where $\boldsymbol{I}$ denotes the identity matrix of appropriate dimension and $\rho$ is a small positive constant, e.g., $\rho=10^{-5}$. The first $N_{B}$ elements of $\widehat{\omega}$ provide an initial estimate for $\boldsymbol{\theta}$, which is denoted as $\widehat{\boldsymbol{\theta}}^{(0)}$. Note that $\widehat{\boldsymbol{\theta}}^{(0)}$ is an unbiased estimate for $\boldsymbol{\theta}$ for sufficiently small $\rho$.

$A L S$ estimation procedure. For $1 \leq \tau \leq \tau_{\max }$, e.g., $\tau_{\max }=$ 4, perform:

a) Given $\widehat{\boldsymbol{\theta}}^{(\tau-1)}$, calculate the LS estimate $\widehat{\boldsymbol{h}}^{(\tau)}$. Specifically, define the regression matrix $\boldsymbol{Q} \in \mathbb{C}^{N \times\left(L_{\text {cir }}+1\right)}$

$$
\boldsymbol{Q}=\left[\begin{array}{cccc}
\widehat{w}_{0} & \widehat{w}_{-1} & \cdots & \widehat{w}_{-L_{\mathrm{cir}}} \\
\vdots & \vdots & \vdots & \vdots \\
\widehat{w}_{k} & \widehat{w}_{k-1} & \cdots & \widehat{w}_{k-L_{\mathrm{cir}}} \\
\vdots & \vdots & \vdots & \vdots \\
\widehat{w}_{N-1} & \widehat{w}_{N-2} & \cdots & \widehat{w}_{N-1-L_{\mathrm{cir}}}
\end{array}\right]
$$

in which

$$
\widehat{w}_{k}=\widehat{\Psi}\left(x_{k}\right)=\sum_{l=1}^{N_{R}} \sum_{m=1}^{N_{I}} B_{l, m}^{\left(P_{o}\right)}\left(x_{k}\right) \widehat{\theta}_{l, m}^{(\tau-1)} .
$$

The LS estimate $\widehat{\boldsymbol{h}}^{(\tau)}$ is readily given by

$$
\begin{aligned}
\underline{\widehat{\boldsymbol{h}}}^{(\tau)} & =\left(\boldsymbol{Q}^{\mathrm{H}} \boldsymbol{Q}\right)^{-1} \boldsymbol{Q}^{\mathrm{H}} \boldsymbol{y}, \\
\widehat{h}_{i}^{(\tau)} & =\widehat{\widehat{h}}_{i}^{(\tau)} / \widehat{\hat{h}}_{0}^{(\tau)}, 0 \leq i \leq L_{\mathrm{cir}} .
\end{aligned}
$$

b) Given $\widehat{\boldsymbol{h}}^{(\tau)}$, calculate the LS estimate $\widehat{\boldsymbol{\theta}}^{(\tau)}$. Specifically, introduce

$$
\varphi_{l, m}(k)=\sum_{i=0}^{L_{\mathrm{cir}}} \widehat{h}_{i}^{(\tau)} B_{l, m}^{\left(P_{o}\right)}\left(x_{k-i}\right) \in \mathbb{C} .
$$

Further define the regression matrix

$$
\boldsymbol{S}=[\boldsymbol{\varphi}(0) \boldsymbol{\varphi}(1) \cdots \varphi(N-1)]^{\mathrm{T}} \in \mathbb{C}^{N \times N_{B}},
$$

with $\varphi(k)=\left[\varphi_{1,1}(k) \varphi_{1,2}(k) \cdots \varphi_{l, m}(k) \cdots \varphi_{N_{R}, N_{I}}(k)\right]^{\mathrm{T}}$. The LS estimate $\widehat{\boldsymbol{\theta}}^{(\tau)}$ is given by $\widehat{\boldsymbol{\theta}}^{(\tau)}=\left(\boldsymbol{S}^{\mathrm{H}} \boldsymbol{S}\right)^{-1} \boldsymbol{S}^{\mathrm{H}} \boldsymbol{y}$.

Clearly, this ALS procedure guarantees to converge to the joint unbiased estimate of $\boldsymbol{h}$ and $\boldsymbol{\theta}$ that is the unique minimum solution of the cost function (33). This is simply because given the unbiased estimate $\widehat{\boldsymbol{\theta}}^{(\tau-1)}$ of $\boldsymbol{\theta}$, the LS estimate $\widehat{\boldsymbol{h}}^{(\tau)}$ is the unbiased estimate of $\boldsymbol{h}$, and given the unbiased estimate $\widehat{\boldsymbol{h}}^{(\tau)}$, the LS estimate $\widehat{\boldsymbol{\theta}}^{(\tau)}$ is the unbiased estimate of $\boldsymbol{\theta}$.

Remark 1: Because the B-spline modeling has the optimal robustness property as discussed in Subsection III-D, we expect that the CV B-spline based estimate $\widehat{\Psi}_{B}(x)$ is a more accurate estimate of the true HPA's nonlinearity $\Psi(x)$ than the CV polynomial based estimate $\widehat{\Psi}_{P}(x)$. This will be verified in our comparative performance evaluation. 


\section{F. Inverting HPA's nonlinearity}

1) CV B-spline inverting model: We utilize another Bspline neural network to model the inverse mapping of the HPA's CV nonlinearity defined by

$$
x_{k}=\Psi^{-1}\left(w_{k}\right)=\Phi\left(w_{k}\right) .
$$

Define two knots sequences similar to (25) for $w_{R}$ and $w_{I}$, respectively. We can construct the inverting B-spline model

$$
\begin{aligned}
\widehat{x} & =\widehat{\Phi}_{B}\left(w ; \boldsymbol{\alpha}_{B}\right) \\
& =\sum_{l=1}^{N_{R}} \sum_{m=1}^{N_{I}} B_{l}^{\left(R, P_{o}\right)}\left(w_{R}\right) B_{m}^{\left(I, P_{o}\right)}\left(w_{I}\right) \alpha_{l, m}^{B},
\end{aligned}
$$

where $B_{l}^{\left(R, P_{o}\right)}\left(w_{R}\right)$ and $B_{m}^{\left(I, P_{o}\right)}\left(w_{I}\right)$ are similarly calculated based on De Boor recursion (26) and (27), while

$$
\boldsymbol{\alpha}_{B}=\left[\begin{array}{lll}
\alpha_{1,1}^{B} & \alpha_{1,2}^{B} \cdots \alpha_{l, m}^{B} \cdots \alpha_{N_{R}, N_{I}}^{B}
\end{array}\right]^{\mathrm{T}} \in \mathbb{C}^{N_{B}} .
$$

Inverting the HPA's nonlinearity becomes the problem of estimating $\boldsymbol{\alpha}_{B}$.

2) $C$ Volynomial inverting model: By defining the two sets of polynomial basis functions similar to (30) for $w_{R}$ and $w_{I}$, respectively, we can construct the inverting polynomial model

$$
\begin{aligned}
\widehat{x} & =\widehat{\Phi}_{P}\left(w ; \boldsymbol{\alpha}_{P}\right) \\
& =\sum_{l=0}^{P_{o}} \sum_{m=0}^{P_{o}} P_{l}^{(R)}\left(w_{R}\right) P_{m}^{(I)}\left(w_{I}\right) \alpha_{l, m}^{P},
\end{aligned}
$$

where

$$
\boldsymbol{\alpha}_{P}=\left[\alpha_{0,0}^{P} \alpha_{0,1}^{P} \cdots \alpha_{l, m}^{P} \cdots \alpha_{P_{o}, P_{o}}^{P}\right]^{\mathrm{T}} \in \mathbb{C}^{N_{P}} .
$$

\begin{tabular}{|c|c|c|c|c|c|}
\hline \multirow{2}{*}{$\begin{array}{l}\text { Tap } \\
\text { No. }\end{array}$} & \multirow{2}{*}{$\begin{array}{c}\text { True } \\
\text { parameter }\end{array}$} & \multicolumn{2}{|c|}{$E_{\mathrm{x}} / N_{\mathrm{o}}=5 \mathrm{~dB}$} & \multicolumn{2}{|c|}{$E_{\mathrm{x}} / N_{\mathrm{o}}=10 \mathrm{~dB}$} \\
\hline & & average estimate & standard deviation & average estimate & standard deviation \\
\hline \multicolumn{6}{|c|}{$\mathrm{OBO}=3 \mathrm{~dB}$} \\
\hline$h_{0}$ & 1 & 1 & & 1 & \\
\hline$h_{1}$ & $-0.3732-\mathrm{j} 0.6123$ & $-0.3732-\mathrm{j} 0.6122$ & $9.152 \mathrm{e}-4,1.021 \mathrm{e}-3$ & $-0.3732-\mathrm{j} 0.6123$ & $5.147 \mathrm{e}-4,5.744 \mathrm{e}-4$ \\
\hline$h_{2}$ & $0.3584+\mathrm{j} 0.3676$ & $0.3586+\mathrm{j} 0.3676$ & $9.702 \mathrm{e}-4,8.555 \mathrm{e}-4$ & $0.3585+\mathrm{j} 0.3676$ & $5.455 \mathrm{e}-4,4.812 \mathrm{e}-4$ \\
\hline$h_{3}$ & $0.3052+\mathrm{j} 0.2053$ & $0.3052+\mathrm{j} 0.2052$ & $9.278 \mathrm{e}-4,8.596 \mathrm{e}-4$ & $0.3052+\mathrm{j} 0.2052$ & $5.219 \mathrm{e}-4,4.834 \mathrm{e}-4$ \\
\hline$h_{4}$ & $0.2300+j 0.1287$ & $0.2300+\mathrm{j} 0.1286$ & $7.806 \mathrm{e}-4,8.650 \mathrm{e}-4$ & $0.2300+\mathrm{j} 0.1286$ & $4.391 \mathrm{e}-4,4.865 \mathrm{e}-4$ \\
\hline$h_{5}$ & $0.7071+\mathrm{j} 0.7071$ & $0.7070+\mathrm{j} 0.7069$ & $1.161 \mathrm{e}-3,1.178 \mathrm{e}-3$ & $0.7071+\mathrm{j} 0.7070$ & $6.530 \mathrm{e}-4,6.627 \mathrm{e}-4$ \\
\hline$h_{6}$ & $0.6123-\mathrm{j} 0.3732$ & $0.6122-\mathrm{j} 0.3733$ & $1.051 \mathrm{e}-3,1.115 \mathrm{e}-3$ & $0.6122-\mathrm{j} 0.3732$ & $5.913 \mathrm{e}-4,6.271 \mathrm{e}-4$ \\
\hline$h_{7}$ & $-0.3584+\mathrm{j} 0.3676$ & $-0.3583+\mathrm{j} 0.3675$ & $9.100 \mathrm{e}-4,1.056 \mathrm{e}-3$ & $-0.3584+\mathrm{j} 0.3675$ & $5.119 \mathrm{e}-4,5.939 \mathrm{e}-4$ \\
\hline$h_{8}$ & $-0.2053-\mathrm{j} 0.3052$ & $-0.2054-\mathrm{j} 0.3051$ & $9.343 \mathrm{e}-4,9.233 \mathrm{e}-4$ & $-0.2053-\mathrm{j} 0.3051$ & $5.253 \mathrm{e}-4,5.193 \mathrm{e}-4$ \\
\hline$h_{9}$ & $0.1287-\mathrm{j} 0.2300$ & $0.1287-\mathrm{j} 0.2299$ & $8.017 \mathrm{e}-4,8.728 \mathrm{e}-4$ & $0.1287-\mathrm{j} 0.2299$ & $4.508 \mathrm{e}-4,4.908 \mathrm{e}-4$ \\
\hline \multicolumn{6}{|c|}{$\mathrm{OBO}=5 \mathrm{~dB}$} \\
\hline$h_{0}$ & 1 & 1 & & 1 & \\
\hline$h_{1}$ & $-0.3732-\mathrm{j} 0.6123$ & $-0.3731-\mathrm{j} 0.6122$ & $7.385 \mathrm{e}-4,8.198 \mathrm{e}-4$ & $-0.3732-\mathrm{j} 0.6123$ & $4.154 \mathrm{e}-4,4.611 \mathrm{e}-4$ \\
\hline$h_{2}$ & $0.3584+\mathrm{j} 0.3676$ & $0.3586+\mathrm{j} 0.3675$ & $7.687 \mathrm{e}-4,6.879 \mathrm{e}-4$ & $0.3585+\mathrm{j} 0.3675$ & $4.322 \mathrm{e}-4,3.869 \mathrm{e}-4$ \\
\hline$h_{3}$ & $0.3052+\mathrm{j} 0.2053$ & $0.3052+\mathrm{j} 0.2052$ & $7.505 e-4,6.757 e-4$ & $0.3052+\mathrm{j} 0.2053$ & $4.221 \mathrm{e}-4,3.799 \mathrm{e}-4$ \\
\hline$h_{4}$ & $0.2300+\mathrm{j} 0.1287$ & $0.2300+\mathrm{j} 0.1286$ & $6.253 \mathrm{e}-4,6.947 \mathrm{e}-4$ & $0.2300+\mathrm{j} 0.1287$ & $3.517 \mathrm{e}-4,3.907 \mathrm{e}-4$ \\
\hline$h_{5}$ & $0.7071+\mathrm{j} 0.7071$ & $0.7071+\mathrm{j} 0.7069$ & $9.318 \mathrm{e}-4,9.480 \mathrm{e}-4$ & $0.7071+\mathrm{j} 0.7070$ & $5.239 \mathrm{e}-4,5.332 \mathrm{e}-4$ \\
\hline$h_{6}$ & $0.6123-\mathrm{j} 0.3732$ & $0.6121-\mathrm{j} 0.3732$ & $8.424 \mathrm{e}-4,8.854 \mathrm{e}-4$ & $0.6122-\mathrm{j} 0.3732$ & $4.739 \mathrm{e}-4,4.978 \mathrm{e}-4$ \\
\hline$h_{7}$ & $-0.3584+\mathrm{j} 0.3676$ & $-0.3583+\mathrm{j} 0.3675$ & $7.471 \mathrm{e}-4,8.454 \mathrm{e}-4$ & $-0.3584+\mathrm{j} 0.3675$ & $4.202 \mathrm{e}-4,4.754 \mathrm{e}-4$ \\
\hline$h_{8}$ & $-0.2053-\mathrm{j} 0.3052$ & $-0.2053-\mathrm{j} 0.3052$ & $7.568 \mathrm{e}-4,7.381 \mathrm{e}-4$ & $-0.2053-\mathrm{j} 0.3052$ & $4.256 \mathrm{e}-4,4.151 \mathrm{e}-4$ \\
\hline$h_{9}$ & $0.1287-\mathrm{j} 0.2300$ & $0.1287-\mathrm{j} 0.2299$ & $6.476 \mathrm{e}-4,6.922 \mathrm{e}-4$ & $0.1287-\mathrm{j} 0.2299$ & $3.641 \mathrm{e}-4,3.892 \mathrm{e}-4$ \\
\hline
\end{tabular}

Inverting the HPA's nonlinearity is turned into the problem of estimating $\boldsymbol{\alpha}_{P}$.

TABLE III

IDENTIFICATION RESULTS AVERAGED OVER 100 RUNS FOR THE CIR COEFFICIENT VECTOR $\boldsymbol{h}$ OF THE HAMMERSTEIN CHANNEL USING THE CV B-SPLINE NEURAL NETWORK APPROACH.
3) Estimation Algorithm: To estimate $\boldsymbol{\alpha}_{B}$ or $\boldsymbol{\alpha}_{P}$ needs the input-output training data $\left\{w_{k}, x_{k}\right\}$ but $w_{k}$ is unavailable. We adopt the same pseudo training data approach of [2], [3], by replacing $w_{k}$ with its estimate $\widehat{w}_{k}=\widehat{\Psi}_{B}\left(x_{k}\right)$ or $\widehat{w}_{k}=\widehat{\Psi}_{P}\left(x_{k}\right)$ based on the identified HPA's nonlinearity $\widehat{\Psi}_{B}\left(\right.$ ) or $\widehat{\Psi}_{P}()$.

Again we present the estimation algorithm for the CV Bspline inverting model (44), and drop the subscript $B$ and superscript ${ }^{B}$, since the estimation algorithm for the $\mathrm{CV}$ polynomial inverting model is exactly the same. Over the pseudo training data set $\left\{\widehat{w}_{k}, x_{k}\right\}_{k=0}^{N-1}$, the regression matrix $\boldsymbol{B} \in \mathbb{R}^{N \times N_{B}}$ can be formed as

$$
\boldsymbol{B}=\left[\begin{array}{cccc}
B_{1,1}^{\left(P_{o}\right)}\left(\widehat{w}_{0}\right) & B_{1,2}^{\left(P_{o}\right)}\left(\widehat{w}_{0}\right) & \cdots & B_{N_{R}, N_{I}}^{\left(P_{o}\right)}\left(\widehat{w}_{0}\right) \\
B_{1,1}^{\left(P_{o}\right)}\left(\widehat{w}_{1}\right) & B_{1,2}^{\left(P_{o}\right)}\left(\widehat{w}_{1}\right) & \cdots & B_{N_{R}, N_{I}}^{\left(P_{o}\right)}\left(\widehat{w}_{1}\right) \\
\vdots & \vdots & \vdots & \vdots \\
B_{1,1}^{\left(P_{o}\right)}\left(\widehat{w}_{N-1}\right) & B_{1,2}^{\left(P_{o}\right)}\left(\widehat{w}_{N-1}\right) & \cdots & B_{N_{R}, N_{I}}^{\left(P_{o}\right)}\left(\widehat{w}_{N-1}\right)
\end{array}\right],
$$

and the LS solution is given by $\widehat{\boldsymbol{\alpha}}=\left(\boldsymbol{B}^{\mathrm{T}} \boldsymbol{B}\right)^{-1} \boldsymbol{B}^{\mathrm{T}} \boldsymbol{x}$.

Remark 2: Because the pseudo training input data $\left\{\widehat{w}_{k}\right\}_{k=0}^{N-1}$ are highly noisy, which will seriously affect the polynomial model but not the B-spline model as analyzed in Subsection III-D, the CV polynomial inverting model (46) will be a far less accurate estimate of the true HPA's inversion $\Psi^{-1}()$, compared to the CV B-spline inverting model (44). This will be confirmed by our comparative performance evaluation presented in the next section.

TABLE IV

KNOT SEQUENCES FOR B-SPLINE MODEL AND INVERSE MODEL. Knot sequence for $x_{R}$ and $x_{I}$

$-10.0,-9.0,-1.0,-\mathbf{0 . 9},-0.06,-0.04,0.0,0.04,0.06, \mathbf{0 . 9}, 1.0,9.0,10.0$

Knot sequence for $w_{R}$ and $w_{I}$

$-20.0,-18.0,-3.0,-\mathbf{1 . 4},-0.8,-0.4,0.0,0.4,0.8, \mathbf{1 . 4}, 3.0,18.0,20.0$ 


\section{Comparative Performance Evaluation}

We evaluated the comparative performance of the $\mathrm{CV}$ B-spline based NIFDDFE and the CV polynomial based NIFDDFE for a 64-QAM Hammerstein channel in which the HPA was described by (6) and (7) with the parameter set given in (8). The dispersive channel had 10 taps $\left(L_{\text {cir }}=9\right)$ whose CIR coefficients are given in Table III. The size of the transmitted data block was $N=2048$. The system's signalto-noise ratio (SNR) was defined as $\mathrm{SNR}=E_{\mathrm{x}} / N_{\mathrm{o}}$, where $E_{\mathrm{x}}$ was the average power of the input signal $x_{k}$ to the HPA and $N_{\mathrm{o}}=2 \sigma_{e}^{2}$.

For the CV B-spline neural network based approach, the piecewise quartic polynomial of $P_{o}=4$ was chosen, and the number of B-spline basis functions was set to $N_{R}=N_{I}=8$, while the knot sequences adopted by the two CV B-spline neural networks for identifying and inverting the HPA's nonlinearity are listed in Table IV. For the CV polynomial modeling based approach, we set the polynomial degree to $P_{o}=4$. All the estimation results were obtained by averaging over 100 random runs.

The effectiveness of the CV B-spline neural network based approach to identify this Hammerstein channel is demonstrated in Table III as well as Figs. 5 and 6. It can be seen from Table III that the identification of the CIR tap vector in the Hammerstein channel was achieved with high precision even under the adverse operational condition of $\mathrm{OBO}=3 \mathrm{~dB}$ and $E_{\mathrm{x}} / N_{\mathrm{o}}=5 \mathrm{~dB}$. Note that under the HPA operational condition of $\mathrm{OBO}=5 \mathrm{~dB}$, the peak amplitude of $\left|x_{k}\right|$ was less less than 0.09 , while under the condition of $\mathrm{OBO}=3 \mathrm{~dB}$, the peak amplitude of $\left|x_{k}\right|$ was less than 0.14 . The results of Figs. 5 and 6 clearly demonstrate the capability of the proposed CV B-spline neural network to accurately model the HPA's nonlinearity, within the HPA's operational input
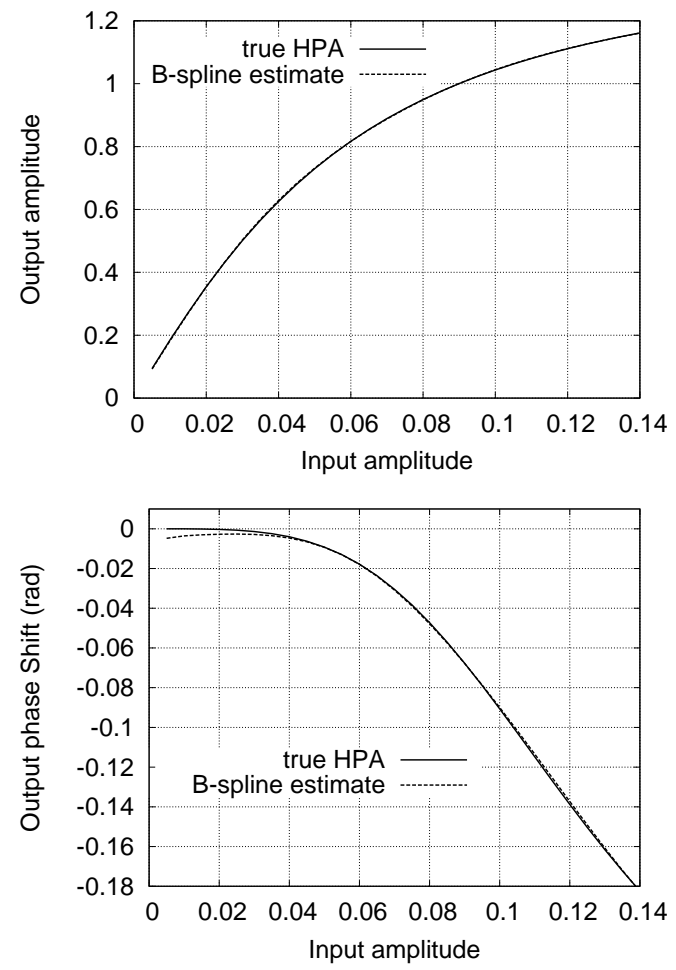

Fig. 5. Comparison of the HPA's nonlinearity $\Psi()$ and its B-spline estimate $\widehat{\Psi}_{B}(\mathrm{l})$ averaged over 100 runs, under $\mathrm{OBO}=3 \mathrm{~dB}$ and $E_{\mathrm{x}} / N_{\mathrm{o}}=5 \mathrm{~dB}$.

range. As a comparison, the results obtained by applying the $\mathrm{CV}$ polynomial based modeling approach to identify this Hammerstein channel are shown in Table $V$ as well as Figs. 7 and 8. Table $\mathrm{V}$ indicates that the linear subsystem of this Hammerstein channel is also identified with high precision by the CV polynomial based approach, which is expected. By comparing Figs. 7 and 8 with Figs. 5 and 6, it can be seen that the CV HPA's nonlinearity identified by the polynomial based

TABLE V

IDENTIFICATION RESULTS AVERAGED OVER 100 RUNS FOR THE CIR COEFFICIENT VECTOR $\boldsymbol{h}$ OF THE HAMMERSTEIN CHANNEL USING THE CV POLYNOMIAL MODELING APPROACH.

\begin{tabular}{|c|c|c|c|c|c|}
\hline \multirow{2}{*}{$\begin{array}{l}\text { Tap } \\
\text { No. }\end{array}$} & \multirow{2}{*}{$\begin{array}{c}\text { True } \\
\text { parameter }\end{array}$} & \multicolumn{2}{|c|}{$E_{\mathrm{x}} / N_{\mathrm{o}}=5 \mathrm{~dB}$} & \multicolumn{2}{|c|}{$E_{\mathrm{x}} / N_{\mathrm{o}}=10 \mathrm{~dB}$} \\
\hline & & average estimate & standard deviation & average estimate & iation \\
\hline \multicolumn{6}{|c|}{$\mathrm{OBO}=3 \mathrm{~dB}$} \\
\hline$h_{0}$ & 1 & 1 & & 1 & \\
\hline$h_{1}$ & $-0.3732-\mathrm{j} 0.6123$ & $-0.3735-\mathrm{j} 0.6120$ & $9.176 \mathrm{e}-4,1.027 \mathrm{e}-3$ & $-0.3735-\mathrm{j} 0.6120$ & $5.160 \mathrm{e}-4,5.778 \mathrm{e}-4$ \\
\hline$h_{2}$ & $0.3584+\mathrm{j} 0.3676$ & $0.3596+\mathrm{j} 0.3680$ & $9.723 \mathrm{e}-4,8.540 \mathrm{e}-4$ & $0.3595+\mathrm{j} 0.3680$ & $5.468 \mathrm{e}-4,4.805 \mathrm{e}-4$ \\
\hline$h_{3}$ & $3052+j 0$ & $0.3052+\mathrm{j} 0.2058$ & $9.262 \mathrm{e}-4,8.591 \mathrm{e}-4$ & $0.3053+\mathrm{j} 0.2059$ & $5.209 \mathrm{e}-4,4.831 \mathrm{e}-4$ \\
\hline$h_{4}$ & $0.2300+\mathrm{j} 0.1287$ & $0.2310+\mathrm{j} 0.1277$ & $7.786 \mathrm{e}-4,8.603 \mathrm{e}-4$ & $0.2310+\mathrm{j} 0.1277$ & $4.379 \mathrm{e}-4,4.837 \mathrm{e}-4$ \\
\hline$h_{5}$ & $0.7071+\mathrm{j} 0.7071$ & $0.7072+\mathrm{j} 0.7066$ & $1.165 \mathrm{e}-3,1.187 \mathrm{e}-3$ & $0.7072+\mathrm{j} 0.7067$ & $6.552 \mathrm{e}-4,6.677 \mathrm{e}-4$ \\
\hline$h_{6}$ & $0.6123-\mathrm{j} 0.3732$ & $0.6118-\mathrm{j} 0.3721$ & $1.052 \mathrm{e}-3,1.116 \mathrm{e}-3$ & $0.6118-\mathrm{j} 0.3721$ & $5.920 \mathrm{e}-4,6.278 \mathrm{e}-4$ \\
\hline$h_{7}$ & $0.3584+\mathrm{j} 0.3676$ & $-0.3582+j 0.3689$ & $9.077 \mathrm{e}-4,1.055 \mathrm{e}-3$ & $-0.3582+\mathrm{j} 0.3689$ & $5.105 \mathrm{e}-4,5.930 \mathrm{e}-4$ \\
\hline$h_{8}$ & $-0.2053-\mathrm{j} 0.3052$ & $-0.2064-\mathrm{j} 0.3052$ & $27 \mathrm{e}-4,9.284 \mathrm{e}-4$ & $-0.2063-\mathrm{j} 0.3052$ & $5.245 \mathrm{e}-4,5.221 \mathrm{e}-4$ \\
\hline$h_{9}$ & $0.1287-\mathrm{j} 0.2300$ & $34-\mathrm{j} 0.2291$ & $8.057 \mathrm{e}-4,8.615 \mathrm{e}-4$ & $0.1284-\mathrm{j} 0.2292$ & $4.531 \mathrm{e}-4,4$. \\
\hline \multicolumn{6}{|c|}{$\mathrm{OBO}=5 \mathrm{~dB}$} \\
\hline$h_{0}$ & 1 & 1 & & 1 & \\
\hline$h_{1}$ & $-0.3732-\mathrm{j} 0.6123$ & $-0.3740-\mathrm{j} 0.6121$ & $7.360 \mathrm{e}-4,8.281 \mathrm{e}-4$ & $-0.3741-\mathrm{j} 0.6121$ & $4.138 \mathrm{e}-4,4.657 \mathrm{e}-4$ \\
\hline$h_{2}$ & $0.3584+\mathrm{j} 0.3676$ & $0.3595+\mathrm{j} 0.3681$ & $7.778 \mathrm{e}-4,6.846 \mathrm{e}-4$ & $0.3594+\mathrm{j} 0.3681$ & $4.374 \mathrm{e}-4,3.851 \mathrm{e}-4$ \\
\hline$h_{3}$ & $0.3052+\mathrm{j} 0.2053$ & $0.3058+\mathrm{j} 0.2058$ & $7.471 \mathrm{e}-4,6.809 \mathrm{e}-4$ & $0.3058+\mathrm{j} 0.2058$ & $4.202 \mathrm{e}-4,3.829 \mathrm{e}-4$ \\
\hline$h_{4}$ & $0.2300+\mathrm{j} 0.1287$ & $0.2310+\mathrm{j} 0.1271$ & $6.298 \mathrm{e}-4,6.991 \mathrm{e}-4$ & $0.2310+\mathrm{j} 0.1272$ & $3.542 \mathrm{e}-4,3.931 \mathrm{e}-4$ \\
\hline$h_{5}$ & $0.7071+\mathrm{j} 0.7071$ & $0.7074+\mathrm{j} 0.7074$ & $9.378 \mathrm{e}-4,9.594 \mathrm{e}-4$ & $0.7074+\mathrm{j} 0.7074$ & $5.273 e-4,5.396 e-4$ \\
\hline$h_{6}$ & $0.6123-\mathrm{j} 0.3732$ & $0.6124-\mathrm{j} 0.3729$ & $8.423 \mathrm{e}-4,8.941 \mathrm{e}-4$ & $0.6125-\mathrm{j} 0.3729$ & $4.737 \mathrm{e}-4,5.028 \mathrm{e}-4$ \\
\hline$h_{7}$ & $-0.3584+\mathrm{j} 0.3676$ & $-0.3583+\mathrm{j} 0.3686$ & $7.338 \mathrm{e}-4,8.443 \mathrm{e}-4$ & $-0.3584+\mathrm{j} 0.3686$ & $4.127 \mathrm{e}-4,4.748 \mathrm{e}-4$ \\
\hline$h_{8}$ & $-0.2053-\mathrm{j} 0.3052$ & $-0.2056-\mathrm{j} 0.3056$ & $7.538 \mathrm{e}-4,7.359 \mathrm{e}-4$ & $-0.2056-\mathrm{j} 0.3056$ & $4.239 \mathrm{e}-4,4.138 \mathrm{e}-4$ \\
\hline$h_{9}$ & $0.1287-\mathrm{j} 0.2300$ & $0.1285-\mathrm{j} 0.2297$ & $6.469 \mathrm{e}-4,6.860 \mathrm{e}-4$ & $0.1285-\mathrm{j} 0.2297$ & $3.638 \mathrm{e}-4,3.858 \mathrm{e}-4$ \\
\hline
\end{tabular}



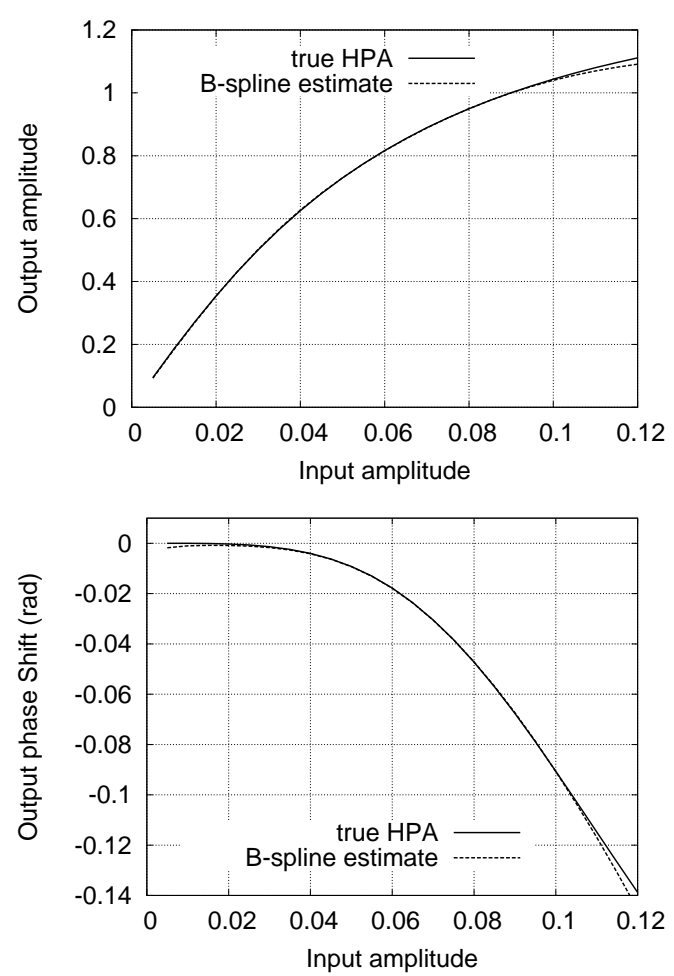

Fig. 6. Comparison of the HPA's nonlinearity $\Psi($ ) and its B-spline estimate $\widehat{\Psi}_{B}\left(\right.$ ) averaged over 100 runs, under $\mathrm{OBO}=5 \mathrm{~dB}$ and $E_{\mathrm{x}} / N_{\mathrm{o}}=10 \mathrm{~dB}$.

approach is less accurate than the B-spline based approach within the HPA's operational input range, which confirms the analysis of Subsection III-D.

The combined responses of the HPA's true nonlinearity and its estimated inversion obtained by the CV B-spline inverting scheme under the two operating conditions are depicted in
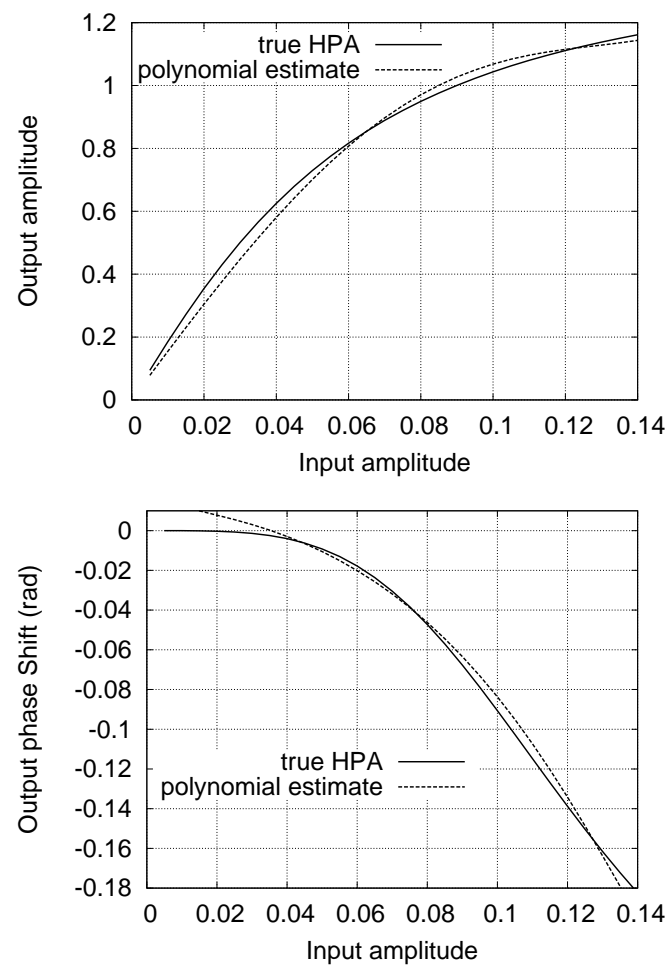

Fig. 7. Comparison of the HPA's nonlinearity $\Psi($ ) and its polynomial estimate $\widehat{\Psi}_{P}()$ averaged over 100 runs, under $\mathrm{OBO}=3 \mathrm{~dB}$ and $E_{\mathrm{x}} / N_{\mathrm{o}}=$ $5 \mathrm{~dB}$.
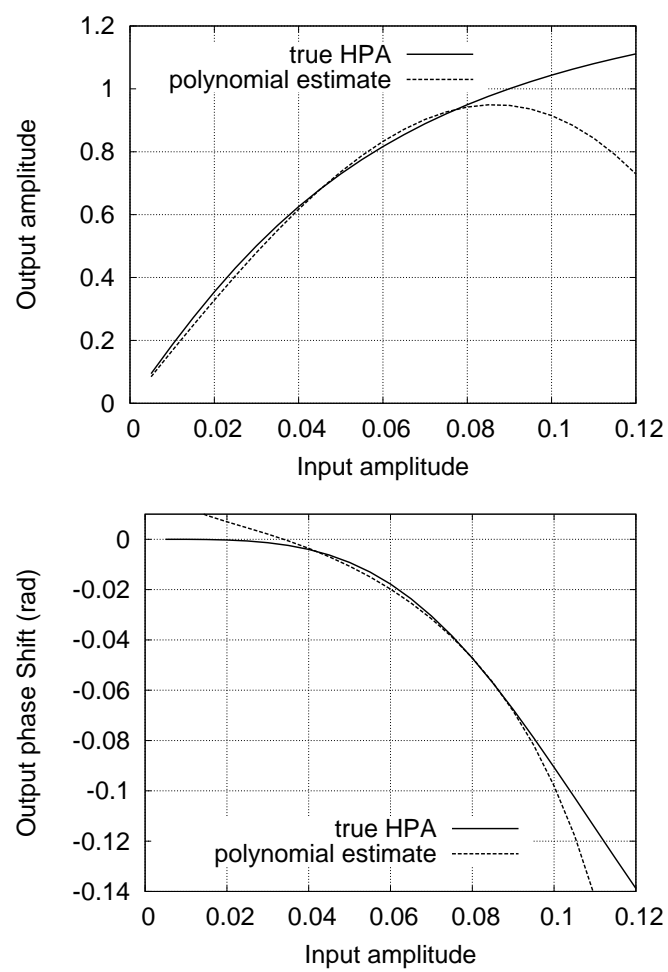

Fig. 8. Comparison of the HPA's nonlinearity $\Psi($ ) and its polynomial estimate $\widehat{\Psi}_{P}()$ averaged over 100 runs, under $\mathrm{OBO}=5 \mathrm{~dB}$ and $E_{\mathrm{x}} / N_{\mathrm{O}}=$ $10 \mathrm{~dB}$.

Figs. 9 and 10. The results clearly show the capability of the CV B-spline neural network to accurately model the inversion of the HPA's nonlinearity based only on the pseudo training data. More specifically, the results of Figs. 9 and 10 clearly indicate that the combined response of the true HPA's nonlinearity $\Psi\left(\right.$ ) and its estimated inversion $\widehat{\Phi}_{B}($ )
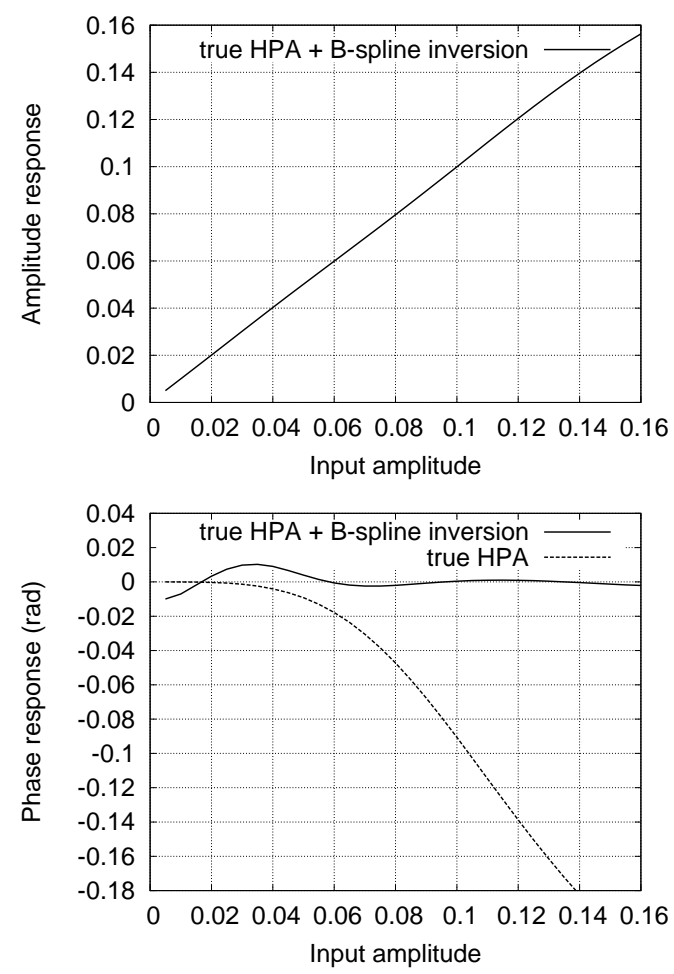

Fig. 9. Combined response of the true HPA and its estimated B-spline inversion averaged over 100 runs, under $\mathrm{OBO}=3 \mathrm{~dB}$ and $E_{\mathrm{x}} / N_{\mathrm{O}}=5 \mathrm{~dB}$. 

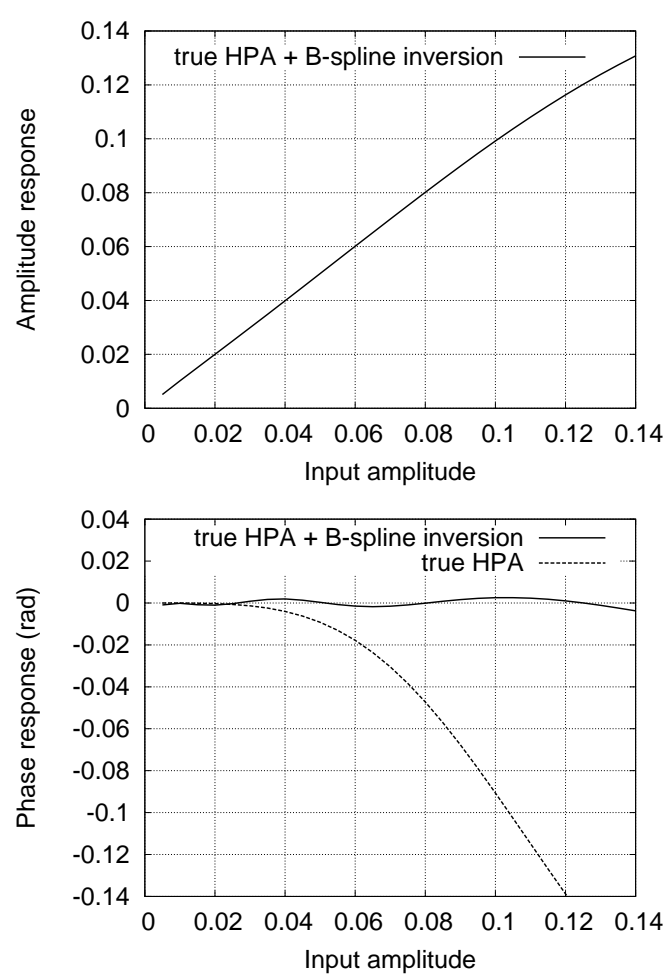

Fig. 10. Combined response of the true HPA and its estimated B-spline inversion averaged over 100 runs, under $\mathrm{OBO}=5 \mathrm{~dB}$ and $E_{\mathrm{x}} / N_{\mathrm{O}}=10 \mathrm{~dB}$.

satisfies $\widehat{\Phi}_{B}(\Psi(x)) \approx x$. That is, the magnitude of the combined response is $\left|\widehat{\Phi}_{B}(\Psi(x))\right| \approx|x|$ and the phase shift of the combined response is approximately zero. In other words, $\widehat{\Phi}_{B}()$ is an accurate inversion of $\Psi($ ). This clearly demonstrates the optimal robustness property of the B-spline modeling presented in Subsection III-D. By contrast, the combined responses of the HPA's true nonlinearity and its
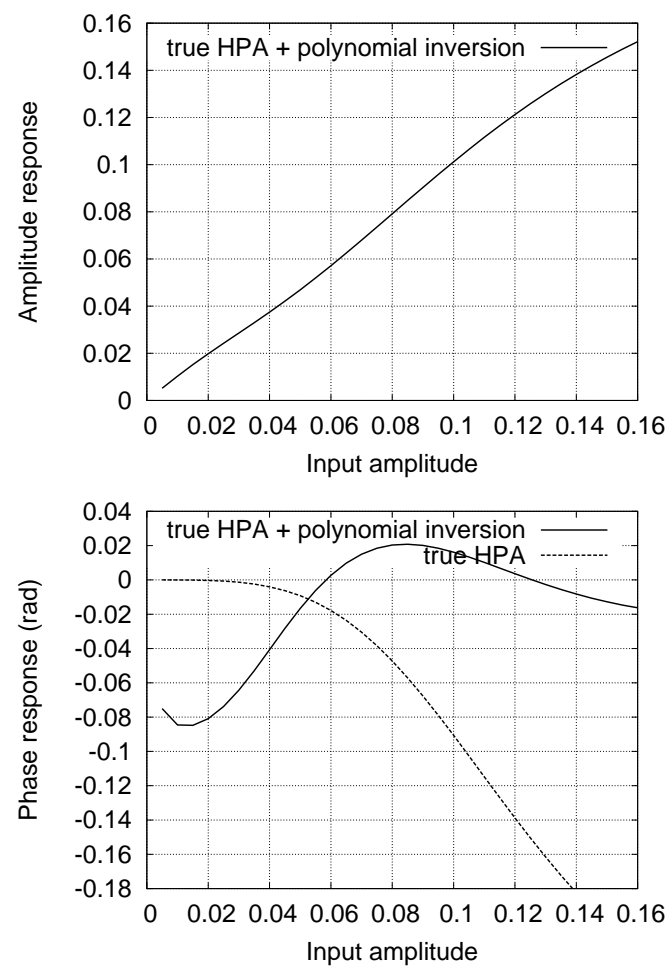

Fig. 11. Combined response of the true HPA and its estimated polynomial inversion averaged over 100 runs, under $\mathrm{OBO}=3 \mathrm{~dB}$ and $E_{\mathrm{x}} / N_{\mathrm{O}}=5 \mathrm{~dB}$.
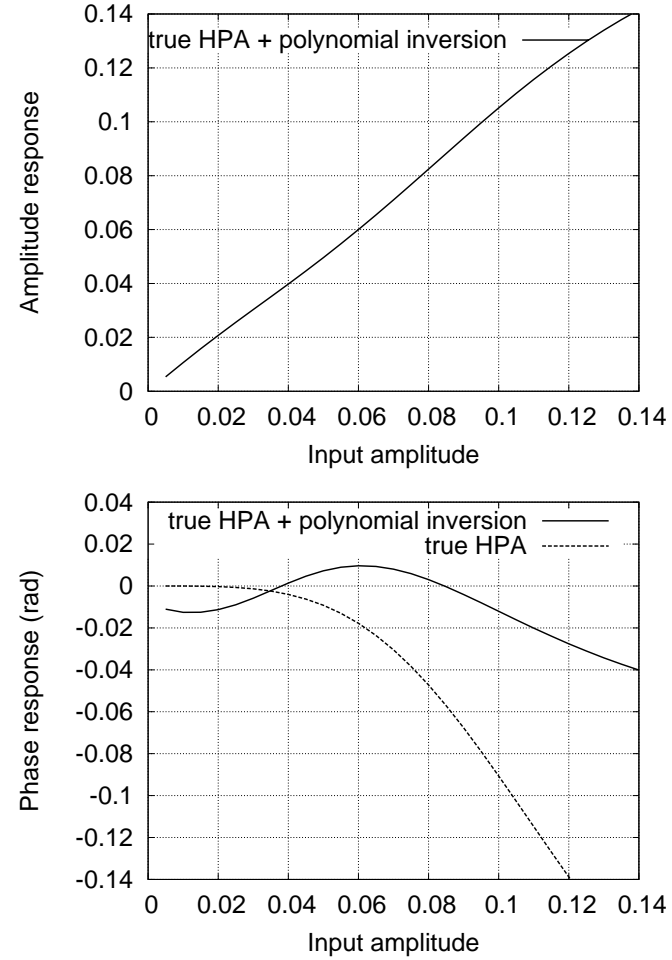

Fig. 12. Combined response of the true HPA and its estimated polynomial inversion averaged over 100 runs, under $\mathrm{OBO}=5 \mathrm{~dB}$ and $E_{\mathrm{x}} / N_{\mathrm{O}}=10 \mathrm{~dB}$.

estimated polynomial inversion depicted in Figs. 11 and 12 under the two HPA operating conditions unmistakably show that the polynomial based inversion estimate $\widehat{\Phi}_{P}()$ is much less accurate than the B-spline based estimate. Evidently, the polynomial modeling is much more sensitive to the noise contained in the pseudo training input $\left\{\widehat{w}_{k}\right\}$.

The bit error rate (BER) performance of the B-spline based NIFDDFE constructed using the estimated CIR, HPA and HPA's inversion are plotted in Fig. 13 under the two HPA operating conditions. From Fig. 13, it can be seen that four iterations are sufficient for the NIFDDFE. Since the first iteration of the NIFDDFE is identical to the NFDE solution without using decision feedback [3], the results of Fig. 10 confirm that the NIFDDFE significantly outperforms the NFDE. The BER performance of the polynomial based NIFDDFE again constructed using the estimated CIR, HPA and HPA's inversion

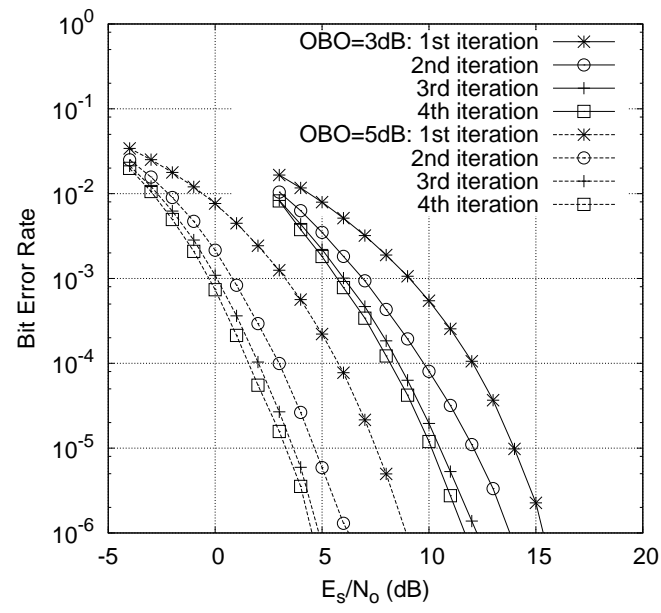

Fig. 13. BER performance of the B-spline based NIFDDFE under the two $\mathrm{HPA}$ operating conditions of $\mathrm{OBO}=3 \mathrm{~dB}$ and $\mathrm{OBO}=5 \mathrm{~dB}$. 


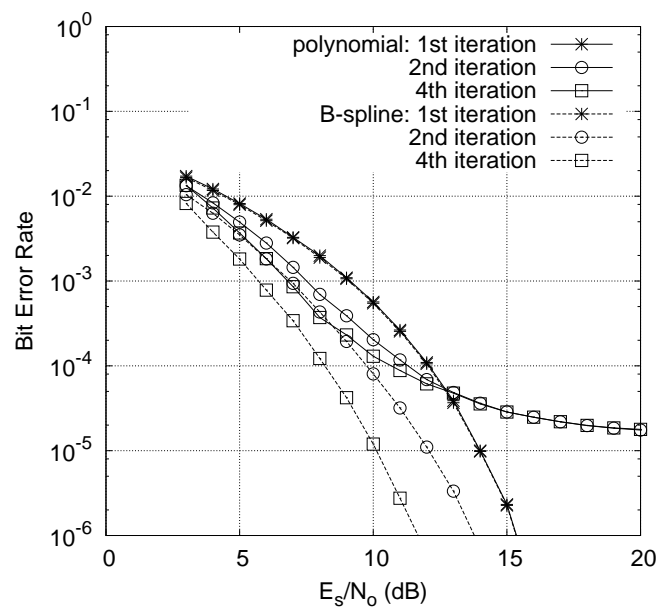

(a) $\mathrm{OBO}=3 \mathrm{~dB}$

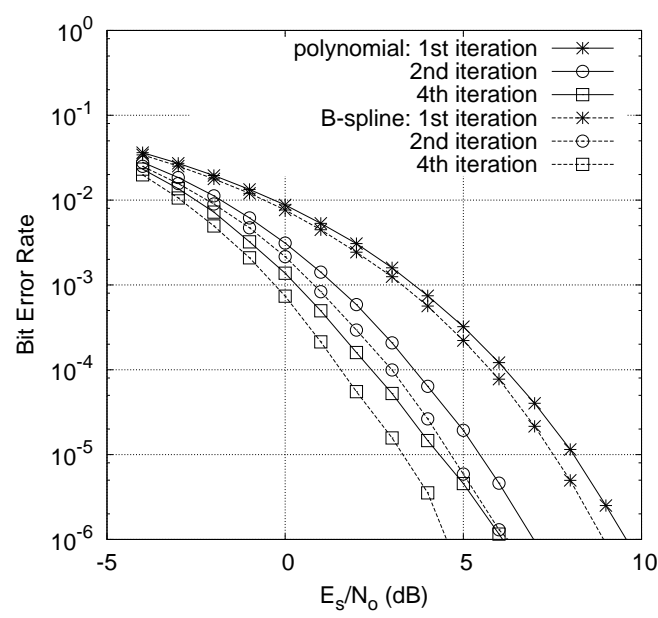

(a) $\mathrm{OBO}=5 \mathrm{~dB}$

Fig. 14. BER performance comparison of the B-spline based NIFDDFE and the polynomial based NIFDDFE.

are depicted in Fig. 14, in comparison with the results of the B-spline based NIFDDFE. The results of Fig. 14 clearly demonstrates that the B-spline based NIFDDFE significantly outperforms the polynomial based NIFDDFE. In particular, when the HPA is operating in the severe nonlinear region, the polynomial based NIFDDFE exhibits a high error floor but this is not the case for the B-spline based NIFDDFE.

\section{CONCLUSiOns}

This paper has evaluated comparative performance of the $\mathrm{CV}$ B-spline neural network and polynomial modeling approaches applied to state-of-the-art iterative frequency-domain decision feedback equalization of Hammerstein communication channels with nonlinear HPA at transmitter. The optimal robustness of the B-spline modeling has been reviewed and it has been shown that the CV B-spline modeling approach has a comparable computational complexity to the conventional CV polynomial modeling approach. Simulation results obtained have verified that the CV B-spline based NIFDDFE significantly outperforms the CV polynomial based NIFDDFE design of comparable complexity. Our conclusions have thus demonstrated that the CV B-spline neural network approach offers a highly effective and accurate means for identifying and inverting Hammerstein systems.

\section{REFERENCES}

[1] S. Chen, X. Hong, J. B. Gao, and C. J. Harris, "Complex-valued B-spline neural networks for modeling and inverting Hammerstein systems," IEEE Trans. Neural Networks and Learning Systems, vol. 25, no. 9, pp. 1673-1685, Sep. 2014.

[2] X. Hong, S. Chen, and C. J. Harris, "B-spline neural network based single-carrier frequency domain equalisation for Hammerstein channels," in Proc. 2014 IJCNN (Beijing, China), Jul. 6-11, 2014, pp. 1834-1841.

[3] X. Hong, S. Chen, C. J. Harris, and E. Khalaf, "Single-carrier frequency domain equalization for Hammerstein communication systems using complex-valued neural networks," IEEE Trans. Signal Proces., vol. 62, no. 17 , pp. 4467-4478, 2014.

[4] J. M. Pena, "B-spline and optimal stability," Mathematics of Computation, vol. 66, no. 220, pp. 1555-1560, Oct. 1997.

[5] T. Lyche and J. M. Pena, "Optimally stable multivariate bases," Advances in Computational Mathematics, vol. 20, nos. 1-3, pp. 149-159, Jan. 2004.

[6] E. Mainar and J. M. Pena, "Optimal stability of bivariate tensor product B-bases," J. Numerical Analysis, Industrial and Applied Mathematics, vol. 6 nos. 3-4, pp. 95-104, 2011.

[7] D. Falconer, S. L. Ariyavisitakul, A. Benyamin-Seeyar, and B. Eidson, "Frequency domain equalization for single carrier broadband wireless systems," IEEE Commun. Mag., vol. 40, no. 4, pp. 58-66, Apr. 2002.

[8] N. Benvenuto and S. Tomasin, "Iterative design and detection of a DFE in the frequency domain," IEEE Trans. Commun., vol. 53, no. 11, pp. 1867-1875, Nov. 2005.

[9] N. Benvenuto, R. Dinis, F. Falconer, and S. Tomasin, "Single carrier modulation with nonlinear frequency domain equalization: An idea whose time has come - again," Proc. IEEE, vol. 98, no, 1, pp. 69-96, Jan. 2010.

[10] C. Zhang, Z. Wang, C. Pan, S. Chen, and L. Hanzo, "Low-complexity iterative frequency domain decision feedback equalization," IEEE Trans. Vehicular Technology, vol. 60, no. 3, pp.1295-1301, Mar. 2011.

[11] M. Honkanen and S.-G. Häggman, "New aspects on nonlinear power amplifier modeling in radio communication system simulations," in Proc. PIMRC'97 (Helsinki, Finland), Sept. 1-4, 1997, pp. 844-848.

[12] C.-S. Choi, et al.,"RF impairment models $60 \mathrm{GHz}$ band SYS/PHY simulation," Document IEEE 802.15-06-0477-01-003c, Nov. 2006. https://mentor.ieee.org/802.15/dcn/06/15-06-0477 -01-003c-rf-impairment-models-60ghz-band-sysphy -simulation.pdf

[13] V. Erceg, et al., "60 GHz impairments modeling," Document IEEE 802.11-09/1213r1, Nov. 2009.

[14] C. De Boor, A Practical Guide to Splines. New York: Spring Verlag, 1978.

[15] C. J. Harris, X. Hong, and Q. Gan, Adaptive Modelling, Estimation and Fusion from Data: A Neurofuzzy Approach. Berlin: Springer-Verlag, 2002.

[16] S. Chen, X. Hong, E. Khalaf, A. Morfeq, and N. Alotaibi, "Adaptive Bspline neural network based nonlinear equalization for high-order QAM systems with nonlinear transmit high power amplifier," Digital Signal Processing, vol. 40, pp. 238-249, May 2015.

[17] A. V. Ivanov, "An asymptotic expansion for the distribution of the least squares estimator of the non-linear regression parameter," SIAM Theory of Probability \& Its Applications, vol. 21, no. 3, pp. 557-570, 1977.

[18] C.-F. Wu, "Asymptotic theory of nonlinear least squares estimation," Annals of Statistics, vol. 9, no. 3, pp. 501-513, 1981. 


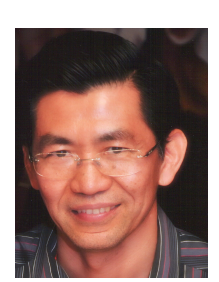

Sheng Chen (M'90-SM'97-F'08) received his BEng degree from the East China Petroleum Institute, Dongying, China, in 1982, and his $\mathrm{PhD}$ degree from the City University, London, in 1986, both in control engineering. In 2005, he was awarded the higher doctoral degree, Doctor of Sciences (DSc), from the University of Southampton, Southampton, UK.

From 1986 to 1999, He held research and academic appointments at the Universities of Sheffield, Edinburgh and Portsmouth, all in UK. Since 1999, he has been with Electronics and Computer Science, the University of Southampton, UK, where he currently holds the post of Professor in Intelligent Systems and Signal Processing. Dr Chen's research interests include adaptive signal processing, wireless communications, modelling and identification of nonlinear systems, neural network and machine learning, intelligent control system design, evolutionary computation methods and optimisation. He has published over 550 research papers.

Dr. Chen is a Fellow of the United Kingdom Royal Academy of Engineering, a Fellow of IET, a Distinguished Adjunct Professor at King Abdulaziz University, Jeddah, Saudi Arabia, and an ISI highly cited researcher in engineering (March 2004).

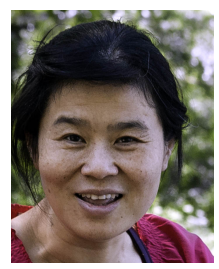

Xia Hong (SM'02) received her university education at National University of Defense Technology, P. R. China (BSc, 1984, MSc, 1987), and University of Sheffield, UK (PhD,1998), all in automatic control. She worked as a research assistant in Beijing Institute of Systems Engineering, Beijing, China from 1987-1993. She worked as a research fellow in the Department of Electronics and Computer Science at University of Southampton from 1997-2001. She is currently a Professor at Department of Computer Science, School of Mathematical, Physical and

Computational Sciences, University of Reading. She is actively engaged in research into nonlinear systems identification, data modelling, estimation and intelligent control, neural networks, pattern recognition, learning theory and their applications. She has published over 100 research papers, and coauthored a research book.

Professor Hong was awarded a Donald Julius Groen Prize by IMechE in 1999.

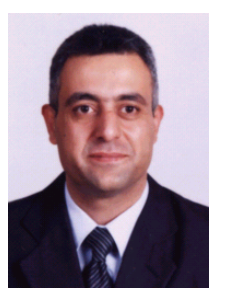

Emad F. Khalaf received his BEng and MEng degrees in IT from Wroclaw University of Technology in Poland, in 1992, as one certificate, and the $\mathrm{PhD}$ degree in Computer networks from Wroclaw University of Technology, in Poland, in 2002.

From 2003 to 2011, he worked as an assistant professor at Computer Engineering Department, Faculty of Engineering, Philadelphia University, in Jordan. Since 2012 he is an assistant professor at Electrical and Computer Engineering Department, Faculty of Engineering, King Abdulaziz University, Jeddah, Saudi Arabia. Dr Khalaf's research interests are in network security and cryptography, speech classification and recognition.
Fuad E. Alsaadi received the B.S. and M.Sc. degrees in electronic and communication from King Abdulaziz University, Jeddah, Saudi Arabia, in 1996 and 2002, respectively. He received the Ph.D. degree in optical wireless communication systems from the University of Leeds, Leeds, UK, in 2011.

Between 1996 and 2005, he worked in Jeddah as a communication instructor in the College of Electronics \& Communication. He was a lecturer in the Faculty of Engineering in King Abdulaziz University, Jeddah, Saudi Arabia in 2005. He is currently an associate professor of the Electrical and Computer Engineering Department within the Faculty of Engineering, King Abdulaziz University, Jeddah, Saudi Arabia.

Dr Alsaadi published widely in the top IEEE communications conferences and journals and received the Carter award from University of Leeds for the best $\mathrm{PhD}$. He has research interests in optical systems and networks, signal processing, network optimization, synchronization and systems design and control.

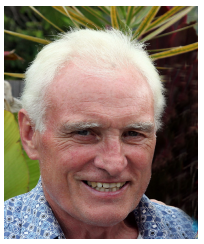

Chris J. Harris received his BSc and MA degrees from the University of Leicester and the University of Oxford in UK, respectively, and his PhD degree from the University of Southampton, UK, in 1972. He was awarded the higher doctoral degree, the Doctor of Sciences (DSc), by the University of Southampton in 2001.

$\mathrm{He}$ is Emeritus Reseach Professor at the University of Southampton, having previously held senior academic appointments at Imperial College, Oxford and Manchester Universities, as well as Deputy Chief Scientist for the UK Government.

Professor Harris was awarded the IEE senior Achievement Medal for Data Fusion research and the IEE Faraday Medal for distinguished international research in Machine Learning. He was elected to the UK Royal Academy of Engineering in 1996. He is the co-author of over 450 scientific research papers during a 45 year research career. 